\title{
Investigation of biopharmaceutical and physicochemical drug properties suitable for orally disintegrating tablets
}

\author{
Asami Ono*, Takumi Tomono ${ }^{1}$, Takuo Ogihara ${ }^{1}$, Katsuhide Terada ${ }^{2, a}$, and Kiyohiko \\ Sugano ${ }^{2}$
}

Asahi Kasei Pharma, 632-1 Mifuku, Izunokuni, Shizuoka 410-2321, Japan.

${ }^{1}$ Laboratory of Clinical Pharmacokinetics, Graduate School of Pharmaceutical Sciences, Takasaki University of Health and Welfare, 60 Nakaorui Takasaki, Gunma 370-0033, Japan.

${ }^{2}$ Department of pharmaceutics, Faculty of pharmaceutical sciences, Toho University, 2-2-1 Miyama, Funabashi, Chiba 274-8510, Japan.

${ }^{a}$ Present address: Laboratory of Molecular Pharmaceutics and Technology, Faculty of Pharmacy, Takasaki University of Health and Welfare, 60 Nakaorui Takasaki, Gunma 370-0033, Japan.

*Corresponding Author: E-mail: ono.ar@om.asahi-kasei.co.jp; Tel.: +81-558-76-7061; Fax: +81-558-76-7137

Received: September 14, 2016; Revised: November 18, 2016; Published: December 26, 2016

\begin{abstract}
The purpose of this study was to evaluate the biopharmaceutical and physicochemical drug properties suitable for orally disintegrating tablets (ODTs). The molecular weight (MW), polar surface area (PSA), hydrogen bond donor (HBD) and acceptor (HBA) numbers, net charge at $p H 7.4, \log D_{6.5}$, the highest dose strength, solubility in water, dose number, and elimination $t_{1 / 2}$ of 57 ODT drugs and 113 drugs of immediate-release (IR) formulations were compared. These drugs were classified according to the Biopharmaceutical Classification System (BCS). A lower dose strength and a longer elimination $t_{1 / 2}$ have been observed as characteristic properties of ODTs. The proportion of basic drugs was higher in the ODTs than in the IR formulations. A significant difference was not observed between the ODT and the IR formulation for $M W, P S A, H B D, H B A$, log $D_{6.5}$, solubility in water, and dose number. The distributions of the ODTs and IR formulations among each BCS class were similar, suggesting that an ODT can be developed regardless of the BCS class of a drug.
\end{abstract}

\section{Keywords}

orally disintegrating tablet (ODT); bioequivalence; biopharmaceutics classification system (BCS).

\section{Introduction}

An orally disintegrating tablet (ODT) improves patient compliance because it can be taken without water, does not cause dysphagia, and can prevent patients from spitting out their medication [1,2]. Many pharmaceutical companies are working on the development of ODTs. An ODT should be bioequivalent to a corresponding standard formulation, e.g. an immediate-release (IR) formulation. An ODT disintegrates and dissolves rapidly in the oral cavity within 30 seconds. Therefore, the dissolution rate may differ significantly between ODTs and IR formulations. When the dissolution rates are different between the ODT and the IR formulation, the risk to fail in a bioequivalence $(B E)$ study is high. Therefore, during the development of an ODT, it is important to assess the risk of failing in a clinical BE study. It is preferable to reduce the risk of failing a clinical BE study because such studies are expensive, time consuming, and a burden to healthy 
volunteers.

However, there has been no research investigating the biopharmaceutical properties of drugs suitable to be an ODT. In the present study, several physicochemical and biopharmaceutical properties were selected and a survey was performed to compare the properties between the two types of oral dose formulations.

\section{Methods}

\section{Drug list}

The physicochemical and biopharmaceutical properties of drugs marketed as ODTs and IR formulations were compared in this study. Currently, the number of drugs developed as ODT is largest in Japan. In addition, all the ODT formulations approved in Japan were proved to be bioequivalent to the IR formulation with and without water intake. Therefore, the ODT formulations in Japanese market were selected in this study. The list of the ODT drugs was obtained from the Pharmaceuticals and Medicinal Devices Agency website (http://www.info.pmda.go.jp/psearch/html/menu_tenpu_base.html) (Supplement Table 1). The list of the IR formulations were selected from the top 200 pharmaceutical products in Japan (Supplement Table 2) [3]. Finally, 57 compounds for the ODT and 113 compounds for the IR formulation were selected and analysed. The 25 compounds were overlapping between the lists of the ODTs and IR formulations.

\section{Biopharmaceutical drug properties}

The physicochemical and biopharmaceutical properties of drugs related to the formulation design and oral bioavailability were selected $[4,5]$. The molecular weight (MW), polar surface area (PSA), hydrogen bond donor (HBD) and acceptor (HBA) numbers, dissociation constants $\left(p K_{\mathrm{a}}\right)$, and n-octanol/water distribution coefficients at pH6.5 $\left(\log D_{6.5}\right)$ were calculated using ACD Percepta (ACD/Labs Software V 14.0.0 (http://www.acdlabs.com/products/percepta/predictors.php)). The calculated $\mathrm{p} K_{\mathrm{a}}$ and $\log D$ were used for all drugs, as the experimental values were not available for some drugs.

Net charge (NC) at pH 7.4 was represented as the weighted sum of the charge of each species.

$$
\begin{aligned}
& \mathrm{NC}=( \pm 0) \cdot f_{0}+(+1) \cdot f_{+}+(-1) \cdot f_{-}+(+2) \cdot f_{++}+\cdots \\
& \left(f_{0}+f_{+}+f_{-}+f_{++}+\cdots=1\right)
\end{aligned}
$$

where $f_{0}$ is the fraction of the undissociated species, $f_{+}$is that of +1 charged species, etc. Each fraction was calculated by the $\mathrm{p} K_{\mathrm{a}}$ and the $\mathrm{pH}$ (set to be 7.4 in this study) using the Henderson-Hasselbalch equation [6]. The NC equations for acids and bases containing up to three ionization centers are summarized in Supplement Table 3. When a drug was more than $50 \%$ dissociated at pH 7.4, it was classified as an acid (NC $<-0.5$ ) or as a base (NC >0.5). Log $D$ was calculated at $\mathrm{pH} 6.5$ to estimate the permeability, whereas NC was calculated at $\mathrm{pH} 7.4$ to discuss the pharmacokinetics after the absorption. The solubility in water, the highest dose strength, and the elimination $t_{1 / 2}$ were obtained from the prescription information. When a reliable solubility figure was unavailable, a solubility value was assigned based on the solubility category defined by the Japanese pharmacopeia (Supplement Table 4).

\section{Provisional classification according to the Biopharmaceutical Classification System}

According to the Biopharmaceutical Classification System (BCS), drugs can be categorized into the four classes, i.e. high solubility/high permeability (class I), low solubility/high permeability (class II), high solubility/low permeability (class III), and low solubility/low permeability (class IV). Moreover, BCS class II drugs can be sub-classified into acid (class Ila), base (class IIb), and undissociated drugs (class IIc) [7]. 
In the previous studies, solubility in water and calculated $\log D_{6.5}$ were used as the surrogates of solubility and permeability data to provide provisional BCS class $[3,8]$. The same approach was taken in this study. According to the official BCS guidance, the equilibrium solubility of a drug at the physiological gastrointestinal pH range (namely, $\mathrm{pH} 1.2$ to $\mathrm{pH} 6.8$ or 7.4 ) is required. However, the $\mathrm{pH}$ solubility profile data were not available for many drugs. Therefore, solubility in water reported in the prescription information was used in this study. The dose number (Do) is a dimensionless number expressed by the ratio of the dose and the maximum dissolved amount in the intestine. Do was calculated by using Eq. 2 [5]:

$$
D o=\frac{M}{S \times V}
$$

where $S$ is the solubility of a drug in water, $V$ is the intestinal fluid volume (set to be $250 \mathrm{~mL}$ in this study), and $M$ is the highest dose strength. Drugs were defined as highly soluble when the Do was $\leq 1$.

According to the official BCS guidance, to classify the permeability category, the fraction of a dose absorbed (Fa\%) in humans or Caco-2 permeability data is required. However, due to the limited availability of these data, the permeability was classified based on the calculated $\log D_{6.5}$ in this study. The $\log D_{6.5}$ value of metoprolol was chosen as the criteria for high permeability [Fa\% in human, $95 \%$ [9]; human effective permeability, $1.26 \times 10^{-4} \mathrm{~cm} / \mathrm{s}$ [9]; $\log D_{6.5},-0.92$ (ACD Percepta)].

\section{Statistical analysis}

A student's t-test was used to evaluate the significance of difference between the ODTs and IR formulations in MW, PSA, HBD, $\mathrm{HBA}, \mathrm{NC}$ at $\mathrm{pH} 7.4, \log D_{6.5}$, the highest dose strength, solubility in water, Do, and elimination $t_{1 / 2}$. One-way analysis of variance was used to evaluate the significance of difference between acids, bases, and undissociated drugs in elimination $t_{1 / 2}$ for the ODTs and IR formulations, respectively. A minimum $p$ value of 0.05 was used as the significance level for all tests. Microsoft Excel 2010 (Microsoft) was used for statistical analysis.

\section{Results}

$\mathrm{MW}$ of free form, PSA, HBD, HBA, NC at pH 7.4, $\log \mathrm{D}_{6.5}$, the highest dose strength, solubility in water, Do, and elimination $t_{1 / 2}$ are shown in Table 1 . Some calculated $\mathrm{p} K_{\mathrm{a}}$ and $\log D_{6.5}$ might have a margin of error about 1 log unit (e.g. $\mathrm{p} K_{\mathrm{a}} \mathrm{s}$ of cetirizine (6.7 (B); 7.7 (B) [10]), domperidone (9.0 (B); 7.1 (B) [10]), glimepiride (5.1 (A); 6.2 (A) [11]), log $D_{6.5}$ of famotidine (-2.14; -1.3 [11]), glimepiride (1.51; 3.0 [11]) (calculated values; experimental values).

$\mathrm{NC}$ at $\mathrm{pH} 7.4$ was significantly higher in the ODT than that for the IR formulation $(p=0.02)$ (Figure $1 \mathrm{e})$. The percentages of acid, base, and undissociated drugs for the ODTs were 13,41 and $45 \%$, respectively ( $2 \%$ unclassifiable). The corresponding percentages in the IR formulations were 30,30 and $38 \%$, respectively ( $2 \%$ unclassifiable). The highest dose strength of the ODTs was significantly lower than that of the IR formulations $(p=0.01$ ) (Figure $1 \mathrm{~g}$ ). The medians of the highest dose strength in the ODTs and IR formulations were $10 \mathrm{mg}$ and $30 \mathrm{mg}$, respectively. The maximum values of the highest dose strength in the ODTs and IR formulations were $200 \mathrm{mg}$ and $900 \mathrm{mg}$, respectively (Table 2 ). The elimination $t_{1 / 2}$ of the ODTs tended to be longer than that of the IR formulations $\left(p=0.07\right.$ ) (Figure $1 \mathrm{j}$ ). The medians of $t_{1 / 2}$ in the ODTs and IR formulations were $6.7 \mathrm{~h}$, and $3.3 \mathrm{~h}$, respectively (Table 2). A significant difference was not observed between the ODTs and the IR formulations for MW, PSA, HBD, HBA, log $D_{6.5}$, solubility in water, and Do (Figure 1a, b, c, d, f, h, i).

The distribution of the ODTs and IR formulations among each BCS class were similar (Figure 2). The percentages of BCS class I, class II, class III, and class IV for the ODT were 52, 29, 20 and $0 \%$, respectively 
(2\% unclassifiable). The corresponding percentages for the IR formulation were 48, 30, 16 and $4 \%$, respectively ( $2 \%$ unclassifiable). The distribution of BCS II subclass was also similar (Figure 2 ). The percentages of BCS class Ila, class IIb, and class Ilc for the ODT were 7, 12 and $9 \%$, respectively, whereas the corresponding percentages for the IR formulation were 10,10 and $11 \%$, respectively.
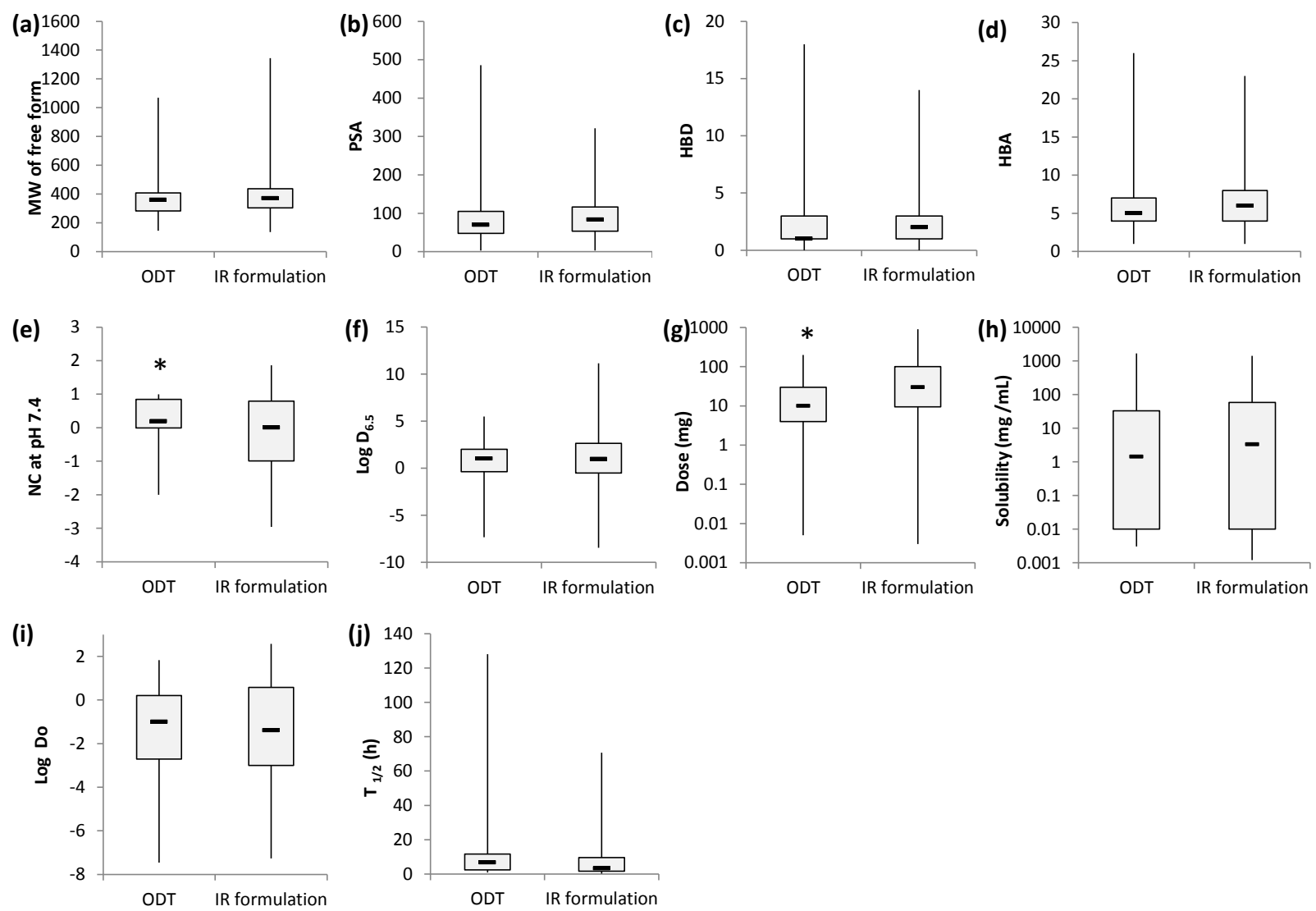

Figure 1. Box and whisker plots of biopharmaceutical properties of ODT and IR formulation drugs ; the bottom and top of the box are the first and third quartiles, the band inside the box is the median, and the ends of the whiskers are the minimum and maximum. (a) MW of free form. (b) Polar surface area. (c) Hydrogen bond donor number. (d) Hydrogen bond acceptor number. (e) Net charge at pH 7.4. (f) Log $D_{6.5}$. (g) The highest dose strength. (h) Solubility in water. (i) Dose number. (j) Elimination $\mathrm{t}_{1 / 2 .}{ }^{*} \mathrm{p}<0.05$. 
Table 1. MW of free form, PSA, HBD, HBA, NC at pH 7.4, $\log D_{6.5}$, the highest dose strength, solubility in water, Do, elimination $t_{1 / 2}$, and BCS class of drugs used in this study.

\begin{tabular}{|c|c|c|c|c|c|c|c|c|c|c|c|c|}
\hline Drug & $\begin{array}{l}\text { MW } \\
\text { (free } \\
\text { form) }\end{array}$ & PSA $^{b}$ & $\mathrm{HBD}^{\mathrm{b}}$ & $\mathrm{HBA}^{\mathrm{b}}$ & $\begin{array}{c}\mathrm{NC}^{\mathrm{a}} \\
\mathrm{pH} 7.4\end{array}$ & $\mathrm{p} K_{\mathrm{a}}{ }^{\mathrm{b}}$ & $\log D_{6.5}^{b}$ & $\begin{array}{l}\text { Highest dose } \\
\text { strength } \\
(\mathrm{mg})^{\mathrm{c}}\end{array}$ & $\begin{array}{l}\text { Solubility } \\
\text { in water } \\
(\mathrm{mg} / \mathrm{mL})^{c}\end{array}$ & Do & $\begin{array}{l}t_{1 / 2} \\
(\mathrm{~h})^{\mathrm{c}}\end{array}$ & $\begin{array}{l}\text { BCS } \\
\text { class }\end{array}$ \\
\hline Acarbose $^{\mathrm{d}, \mathrm{e}}$ & 646 & 321 & 14 & 19 & 0.0 & - & -4.88 & 100 & 1429 & 0.00028 & 3.2 & 3 \\
\hline Acetaminophen & 151 & 49 & 2 & 3 & 0.0 & - & 0.40 & 500 & 10 & 0.2 & 2.9 & 1 \\
\hline Acetylsalicylic acid & 180 & 64 & 1 & 4 & -1.0 & $3.5(A)$ & -1.39 & 100 & 3.33 & 0.12 & 0.4 & 3 \\
\hline Acyclovir & 225 & 115 & 4 & 8 & 0.0 & - & -1.23 & 400 & 1 & 1.6 & 2.4 & 4 \\
\hline Alendronate sodium hydrate & 249 & 181 & 7 & 8 & -2.0 & $\begin{array}{l}1.7(A), 2.7(A) \\
6.1(A), 10.3(B)\end{array}$ & -8.44 & 35 & 25.6 & 0.00546 & 1.5 & 3 \\
\hline Alfacalcidol & 401 & 40 & 2 & 2 & 0.0 & - & 7.58 & 0.03 & 0.01 & 0.0012 & 17.6 & 1 \\
\hline Allopurinol & 136 & 66 & 2 & 5 & 0.0 & - & -0.70 & 100 & 0.1 & 4 & 1.6 & 2 \\
\hline Ambroxol hydrochloride & 378 & 58 & 4 & 3 & 1.0 & $8.7(B)$ & 0.48 & 45 & 26.8 & 0.00672 & 11.2 & 1 \\
\hline Amlodipine besylate & 409 & 100 & 3 & 7 & 1.0 & $9.0(B)$ & 1.09 & 10 & 2.22 & 0.018 & 36.2 & 1 \\
\hline Aripiprazole & 448 & 45 & 1 & 5 & 0.6 & $7.7(B)$ & 4.42 & 24 & 0.01 & 9.6 & 59.6 & 2 \\
\hline Atenolol & 266 & 85 & 4 & 5 & 1.0 & $9.4(B)$ & -2.53 & 50 & 1 & 0.2 & 10.8 & 3 \\
\hline Atorvastatin calcium & 559 & 112 & 4 & 7 & -1.0 & $4.3(A)$ & 2.06 & 10 & 0.145 & 0.276 & 10.8 & 1 \\
\hline Azithromycin hydrate & 749 & 180 & 5 & 14 & 1.8 & $8.2(B), 8.6$ (B) & -0.19 & 600 & 0.01 & 240 & 61.9 & 2 \\
\hline Azulene sulfonate sodium hydrate & 278 & 63 & 1 & 3 & -1.0 & $1.7(A)$ & -2.03 & 2 & 10 & 0.0008 & 2.45 & 3 \\
\hline Benidipine hydrochloride & 506 & 114 & 1 & 9 & 0.8 & $8.0(B)$ & 3.54 & 8 & 0.01 & 3.2 & 1.0 & 2 \\
\hline Bepotastine besylate & 389 & 63 & 1 & 5 & 0.0 & $4.4(A), 8.9(B)$ & 0.86 & 10 & 23.3 & 0.00172 & 2.5 & 1 \\
\hline Beraprost sodium & 398 & 87 & 3 & 5 & -1.0 & $4.8(A)$ & 1.42 & 0.04 & 833 & $1.92 \times 10^{-7}$ & 1.1 & 1 \\
\hline Bicalutamide & 430 & 116 & 2 & 6 & 0.0 & - & 2.53 & 80 & 0.01 & 32 & 4.9 & 2 \\
\hline Bisoprolol fumarate & 325 & 60 & 2 & 5 & 1.0 & $9.4(B)$ & -0.55 & 5 & 1250 & $1.6 \times 10^{-5}$ & 8.6 & 1 \\
\hline Brotizolam & 394 & 71 & 0 & 4 & 0.0 & - & 2.80 & 0.25 & 0.01 & 0.1 & 7 & 1 \\
\hline Cabergoline & 452 & 72 & 2 & 7 & 1.0 & $9.4(B)$ & 0.40 & 1 & 0.01 & 0.4 & 43 & 1 \\
\hline Camostat mesylate & 398 & 137 & 4 & 9 & 1.0 & $9.1(\mathrm{~B})$ & -0.53 & 100 & 45.5 & 0.0088 & 1.7 & 1 \\
\hline Candesartan cilexetil & 440 & 119 & 2 & 9 & -2.0 & $2.1(\mathrm{~A}), 4.2(\mathrm{~A})$ & 0.29 & 12 & 0.01 & 4.8 & 2.2 & 2 \\
\hline
\end{tabular}


Table 1. (Continued)

\begin{tabular}{|c|c|c|c|c|c|c|c|c|c|c|c|c|}
\hline Drug & $\begin{array}{l}\text { MW } \\
\text { (free } \\
\text { form) }\end{array}$ & PSA $^{b}$ & $H_{B D}^{b}$ & $\mathrm{HBA}^{\mathrm{b}}$ & $\begin{array}{c}\mathrm{NC}^{\mathrm{a}} \\
\mathrm{pH} 7.4\end{array}$ & $\mathrm{p} K_{\mathrm{a}}{ }^{\mathrm{b}}$ & $\log D_{6.5}^{b}$ & $\begin{array}{l}\text { Highest dose } \\
\text { strength } \\
(\mathrm{mg})^{c}\end{array}$ & $\begin{array}{l}\text { Solubility } \\
\text { in water } \\
(\mathrm{mg} / \mathrm{mL})^{c}\end{array}$ & Do & $\begin{array}{c}t_{1 / 2} \\
\text { (h) }\end{array}$ & $\begin{array}{l}\text { BCS } \\
\text { class }\end{array}$ \\
\hline Carvedilol & 406 & 76 & 3 & 6 & 0.9 & $8.2(B)$ & 2.20 & 20 & 0.01 & 8 & 7.7 & 2 \\
\hline Cefcapene pivoxil hydrochloride & 594 & 257 & 3 & 16 & 0.0 & $5.9(A), 9.0(B)$ & -1.59 & 100 & 1.88 & 0.213 & 1.1 & 3 \\
\hline Cefdinir & 395 & 212 & 5 & 10 & -1.0 & $2.8(A)$ & -3.92 & 100 & 0.381 & 1.05 & 1.6 & 4 \\
\hline Cefditoren pivoxil & 621 & 257 & 3 & 13 & -0.2 & $8.1(\mathrm{~A})$ & 2.45 & 100 & 0.01 & 40 & 1.1 & 2 \\
\hline Cefotiam hexetil hydrochloride & 696 & 276 & 3 & 16 & 0.8 & $8.0(B)$ & -0.14 & 200 & 1000 & 0.0008 & 0.8 & 1 \\
\hline Cetirizine hydrochloride & 389 & 53 & 1 & 5 & -0.8 & $3.5(A), 6.7(B)$ & -0.09 & 10 & 1000 & $4.0 \times 10^{-5}$ & 7.5 & 1 \\
\hline Cilostazol & 369 & 82 & 1 & 7 & 0.0 & - & 3.01 & 100 & 0.01 & 40 & 10.1 & 2 \\
\hline Clarithromycin & 748 & 183 & 4 & 14 & 0.9 & $8.2(\mathrm{~B})$ & 1.56 & 200 & 0.01 & 80 & 4.4 & 2 \\
\hline Cyclosporin a & 1203 & 279 & 5 & 23 & 0.0 & - & 1.80 & 50 & 3.38 & 0.0592 & 1.6 & 1 \\
\hline Desmopressin acetate hydrate & 1069 & 486 & 18 & 26 & 0.0 & - & -7.34 & 0.24 & 33 & $2.9 \times 10^{-5}$ & 2 & 3 \\
\hline Diclofenac sodium & 296 & 49 & 2 & 3 & -1.0 & $4.2(A)$ & 2.17 & 25 & 17.2 & 0.0058 & 1.2 & 1 \\
\hline Dienogest & 311 & 61 & 1 & 3 & 0.0 & - & 2.64 & 1 & 0.01 & 0.4 & 8.0 & 1 \\
\hline Domperidone & 426 & 68 & 2 & 7 & 1.0 & $9.0(B)$ & 2.26 & 10 & 0.01 & 4 & 0.9 & 2 \\
\hline Donepezil hydrochloride & 379 & 39 & 0 & 4 & 1.0 & $8.8(B)$ & 1.97 & 10 & 10 & 0.004 & 70.7 & 1 \\
\hline Doxazosin mesylate & 451 & 112 & 2 & 10 & 0.1 & $6.5(B)$ & 1.20 & 4 & 1 & 0.016 & 11.8 & 1 \\
\hline Doxifluridine & 246 & 99 & 3 & 7 & -0.4 & $7.6(A)$ & -1.13 & 200 & 33 & 0.0242 & $0.8^{f}$ & 3 \\
\hline Droxidopa & 213 & 124 & 6 & 6 & -0.1 & $2.1(\mathrm{~A}), 8.3(\mathrm{~B})$ & -3.57 & 200 & 2.4 & 0.333 & 2 & 3 \\
\hline Ebastine & 470 & 30 & 0 & 3 & 0.9 & $8.2(B)$ & 5.49 & 10 & 0.01 & 4 & 17.6 & 2 \\
\hline Enalapril maleate & 376 & 96 & 2 & 7 & -1.0 & $3.1(\mathrm{~A})$ & -1.06 & 10 & 21 & 0.00191 & 6.1 & 3 \\
\hline Epalrestat & 319 & 115 & 1 & 4 & -1.0 & $3.6(A)$ & -1.04 & 50 & 0.009 & 22.2 & 1.8 & 4 \\
\hline Eperisone hydrochloride & 259 & 20 & 0 & 2 & 0.9 & $8.5(B)$ & 2.02 & 50 & 200 & 0.001 & 1.6 & 1 \\
\hline Epinastine hydrochloride & 249 & 42 & 2 & 3 & 1.0 & $12.0(\mathrm{~B})$ & 0.77 & 20 & 133 & 0.0006 & 9.2 & 1 \\
\hline Ethyl icosapentate & 331 & 26 & 0 & 2 & 0.0 & - & 6.65 & 900 & 0.01 & 360 & 58.9 & 2 \\
\hline Etizolam & 343 & 71 & 0 & 4 & 0.0 & - & 2.87 & 1 & 0.01 & 0.4 & 6.3 & 1 \\
\hline
\end{tabular}


Table 1. (Continued)

\begin{tabular}{|c|c|c|c|c|c|c|c|c|c|c|c|c|}
\hline Drug & $\begin{array}{l}\text { MW } \\
\text { (free } \\
\text { form) }\end{array}$ & PSA $^{b}$ & $H B D^{b}$ & $\mathrm{HBA}^{\mathrm{b}}$ & $\begin{array}{c}\mathrm{NC}^{\mathrm{a}} \\
\mathrm{pH} 7.4\end{array}$ & $\mathrm{p} K_{\mathrm{a}}^{\mathrm{b}}$ & $\log D_{6.5}{ }^{b}$ & $\begin{array}{l}\text { Highest dose } \\
\text { strength } \\
(\mathrm{mg})^{\mathrm{c}}\end{array}$ & $\begin{array}{l}\text { Solubility } \\
\text { in water } \\
(\mathrm{mg} / \mathrm{mL})^{c}\end{array}$ & Do & $\begin{array}{l}t_{1 / 2} \\
(\mathrm{~h})^{\mathrm{c}}\end{array}$ & $\begin{array}{l}\text { BCS } \\
\text { class }\end{array}$ \\
\hline Famotidine & 337 & 238 & 8 & 9 & 0.8 & 7.9 (B) & -2.14 & 20 & 0.741 & 0.108 & 3.1 & 3 \\
\hline Fexofenadine hydrochloride & 502 & 81 & 3 & 5 & 0.0 & $4.4(A), 9.4(B)$ & 2.43 & 60 & 1.43 & 0.168 & 19 & 1 \\
\hline Fluconazole & 306 & 82 & 1 & 7 & 0.0 & - & 0.70 & 400 & 1 & 1.6 & 30 & 2 \\
\hline Flurbiprofen & 244 & 37 & 1 & 2 & -1.0 & $4.1(A)$ & 1.48 & 40 & 0.01 & 16 & 2.7 & 2 \\
\hline Fluvastatin sodium & 411 & 83 & 3 & 5 & -1.0 & $4.3(A)$ & 1.33 & 30 & 82.0 & 0.00146 & 1.3 & 1 \\
\hline Fluvoxamine maleate & 318 & 57 & 2 & 4 & 1.0 & $9.4(\mathrm{~B})$ & 0.36 & 75 & 14 & 0.0214 & 14.1 & 1 \\
\hline Fursultiamine & 399 & 152 & 3 & 7 & 0.0 & - & 2.05 & 50 & 200 & 0.001 & 14.7 & 1 \\
\hline Galantamine hydrobromide & 287 & 42 & 1 & 4 & 0.8 & 7.9 (B) & 0.12 & 12 & 33 & 0.00145 & 6.8 & 1 \\
\hline Gefitinib & 447 & 69 & 1 & 7 & 0.3 & $7.0(\mathrm{~B})$ & 3.07 & 250 & 0.01 & 100 & 30.1 & 2 \\
\hline Gimeracil & 146 & 53 & 2 & 3 & 0.1 & $6.5(A), 12.6(B)$ & -2.58 & 7.25 & 1.74 & 0.0167 & 3 & 3 \\
\hline Glimepiride & 491 & 133 & 3 & 9 & -1.0 & $5.1(A)$ & 1.51 & 3 & 0.01 & 1.2 & 5.8 & 2 \\
\hline Granisetron hydrochloride & 312 & 50 & 1 & 5 & 1.0 & $10.5(B)$ & -0.95 & 2 & 588 & $1.36 \times 10^{-5}$ & 5.3 & 3 \\
\hline Hydrochlorothiazide & 298 & 135 & 4 & 7 & 0.0 & - & 0.01 & 12.5 & 0.1 & 0.5 & 9.1 & 1 \\
\hline Imatinib mesylate & 494 & 86 & 2 & 8 & 0.6 & $7.6(B)$ & 2.00 & 200 & 1300 & 0.000615 & 15.9 & 1 \\
\hline$\underline{\text { Imidafenacin }}$ & 319 & 61 & 2 & 4 & 0.6 & 7.6 (B) & 1.68 & 0.1 & 0.01 & 0.04 & 3.1 & 1 \\
\hline Imidapril hydrochloride & 405 & 116 & 2 & 9 & -1.0 & $2.4(A)$ & -2.51 & 10 & 49.3 & 0.000812 & 1.7 & 3 \\
\hline Irsogladine maleate & 359 & 96 & 1 & 6 & 0.0 & - & 1.81 & 4 & 0.01 & 1.6 & 128 & 2 \\
\hline Itraconazole & 706 & 101 & 0 & 12 & 0.1 & $6.5(B)$ & 4.67 & 200 & 0.01 & 80 & 27.9 & 2 \\
\hline Ketoprofen & 254 & 54 & 1 & 3 & -1.0 & $4.2(A)$ & 0.87 & 75 & 0.01 & 30 & 1.6 & 2 \\
\hline Ketotifen fumarate & 309 & 49 & 0 & 2 & 1.0 & $8.8(\mathrm{~B})$ & 1.79 & 1 & 1 & 0.004 & 6.7 & 1 \\
\hline L-carbocysteine & 179 & 126 & 4 & 5 & -1.0 & $\begin{array}{c}2.1(\mathrm{~A}), 3.8(\mathrm{~A}) \\
8.8(\mathrm{~B})\end{array}$ & -3.80 & 500 & 0.1 & 20 & 1.6 & 4 \\
\hline$\underline{\text { Lafutidine }}$ & 432 & 104 & 1 & 7 & 0.4 & $7.2(\mathrm{~B})$ & 0.56 & 10 & 0.01 & 4 & 1.6 & 2 \\
\hline Lansoprazole & 369 & 87 & 1 & 5 & 0.0 & - & 2.40 & 30 & 0.0323 & 3.72 & 1.4 & 2 \\
\hline
\end{tabular}


Table 1. (Continued)

\begin{tabular}{|c|c|c|c|c|c|c|c|c|c|c|c|c|}
\hline Drug & $\begin{array}{l}\text { MW } \\
\text { (free } \\
\text { form) }\end{array}$ & PSA $^{b}$ & $H_{B D}^{b}$ & $\mathrm{HBA}^{\mathrm{b}}$ & $\begin{array}{c}\mathrm{NC}^{\mathrm{a}} \\
\mathrm{pH} 7.4\end{array}$ & $\mathrm{p} K_{\mathrm{a}}{ }^{\mathrm{b}}$ & $\log D_{6.5}{ }^{b}$ & $\begin{array}{l}\text { Highest dose } \\
\text { strength } \\
(\mathrm{mg})^{\mathrm{c}}\end{array}$ & $\begin{array}{l}\text { Solubility } \\
\text { in water } \\
(\mathrm{mg} / \mathrm{mL})^{c}\end{array}$ & Do & $\begin{array}{c}t_{1 / 2} \\
\text { (h) }\end{array}$ & $\begin{array}{l}\text { BCS } \\
\text { class }\end{array}$ \\
\hline Levofloxacin & 361 & 73 & 1 & 7 & -0.5 & $5.2(\mathrm{~A}), 7.4(\mathrm{~B})$ & -1.76 & 500 & 16. 7 & 0.12 & 7.9 & 3 \\
\hline Limaprost alfadex & 366 & 95 & 3 & 5 & -1.0 & $4.6(A)$ & 0.85 & 0.005 & 370 & $5.4 \times 10^{-8}$ & 0.5 & 1 \\
\hline Loratadine & 383 & 42 & 0 & 4 & 0.0 & - & 5.32 & 10 & 0.00303 & 13.2 & 20.1 & 2 \\
\hline Losartan potassium & 423 & 93 & 2 & 7 & -1.0 & $4.2(A)$ & 1.77 & 100 & 1000 & 0.0004 & 1.8 & 1 \\
\hline Loxoprofen sodium hydrate & 246 & 54 & 1 & 3 & -1.0 & $4.4(\mathrm{~A})$ & 0.37 & 60 & 1000 & 0.00024 & 1.2 & 1 \\
\hline Manidipine hydrochloride & 611 & 117 & 1 & 10 & 0.1 & $6.1(\mathrm{~B})$ & 5.29 & 20 & 3.88 & 0.0206 & 1.5 & 1 \\
\hline Mecobalamin & 1344 & ND & ND & ND & - & ND & ND & 0.5 & 12.5 & 0.00016 & 12.5 & UC \\
\hline Meloxicam & 351 & 136 & 2 & 7 & -1.0 & $4.5(A)$ & 0.29 & 10 & 0.01 & 4 & 27.6 & 2 \\
\hline Memantine hydrochloride & 179 & 26 & 2 & 1 & 1.0 & 10.8 (B) & 0.40 & 20 & 33 & 0.00242 & 53.6 & 1 \\
\hline Menatetrenone & 445 & 34 & 0 & 2 & 0.0 & - & 9.55 & 15 & 0.01 & 6 & 3.9 & 2 \\
\hline Mesalazine & 153 & 84 & 4 & 4 & -1.0 & $1.9(\mathrm{~A})$ & -1.85 & 500 & 1 & 2 & 6.4 & 4 \\
\hline Methylmethionine sulfonium chloride & 164 & ND & ND & ND & - & ND & ND & 25 & 1000 & 0.0001 & 3.2 & UC \\
\hline Mexiletine hydrochloride & 179 & 35 & 2 & 2 & 0.9 & $8.6(B)$ & 0.39 & 100 & 833 & 0.00048 & 9.4 & 1 \\
\hline Midodrine hydrochloride & 254 & 94 & 4 & 6 & 0.7 & $7.8(B)$ & -1.24 & 2 & 138 & $5.80 \times 10^{-5}$ & 2.4 & 3 \\
\hline Miglitol & 207 & 104 & 5 & 6 & 0.1 & $6.5(B)$ & -2.27 & 75 & 791 & 0.000379 & 2.0 & 3 \\
\hline Montelukast sodium & 586 & 96 & 2 & 4 & -1.0 & $4.8(A)$ & 5.82 & 10 & 200 & 0.0002 & 4.3 & 1 \\
\hline Mosapride citrate hydrate & 422 & 77 & 3 & 6 & 0.1 & $6.2(\mathrm{~B})$ & 2.89 & 5 & 0.01 & 2 & 2 & 2 \\
\hline Naftopizil & 393 & 45 & 1 & 5 & 0.2 & $6.9(\mathrm{~B})$ & 3.65 & 75 & 0.01 & 30 & 11.2 & 2 \\
\hline Nicardipine hydrochloride & 480 & 114 & 1 & 9 & 0.4 & $7.3(\mathrm{~B})$ & 3.94 & 20 & 6 & 0.0133 & 1.5 & 1 \\
\hline Nicergoline & 484 & 57 & 0 & 6 & 0.1 & $6.3(B)$ & 4.29 & 5 & 0.01 & 2 & 3.3 & 2 \\
\hline Nicorandil & 211 & 97 & 1 & 7 & 0.0 & - & 0.93 & 5 & 10 & 0.002 & 0.8 & 1 \\
\hline Nifedipine & 346 & 110 & 1 & 8 & 0.0 & - & 3.45 & 10 & 0.01 & 4 & 1.0 & 2 \\
\hline Nilvadipine & 385 & 134 & 1 & 9 & 0.0 & - & 3.23 & 4 & 0.01 & 1.6 & 10.7 & 2 \\
\hline Nizatidine & 331 & 140 & 2 & 7 & 0.5 & $7.3(\mathrm{~B})$ & -0.61 & 150 & 17.3 & 0.0347 & 1.7 & 1 \\
\hline
\end{tabular}


Table 1. (Continued)

\begin{tabular}{|c|c|c|c|c|c|c|c|c|c|c|c|c|}
\hline Drug & $\begin{array}{l}\text { MW } \\
\text { (free } \\
\text { form) }\end{array}$ & PSA $^{b}$ & $H B D^{b}$ & $\mathrm{HBA}^{\mathrm{b}}$ & $\begin{array}{c}\mathrm{NC}^{\mathrm{a}} \\
\mathrm{pH} 7.4\end{array}$ & $\mathrm{p} K_{\mathrm{a}}^{\mathrm{b}}$ & $\log D_{6.5}^{b}$ & $\begin{array}{l}\text { Highest dose } \\
\text { strength } \\
(\mathrm{mg})^{\mathrm{c}}\end{array}$ & $\begin{array}{l}\text { Solubility } \\
\text { in water } \\
(\mathrm{mg} / \mathrm{mL})^{c}\end{array}$ & Do & $\begin{array}{l}t_{1 / 2} \\
(\mathrm{~h})^{\mathrm{c}}\end{array}$ & $\begin{array}{l}\text { BCS } \\
\text { class }\end{array}$ \\
\hline Olanzapine & 312 & 56 & 1 & 4 & 1.0 & 10.4 (B) & -0.35 & 10 & 0.01 & 4 & 30.6 & 2 \\
\hline Olopatadine hydrochloride & 337 & 50 & 1 & 4 & 0.0 & $4.3(\mathrm{~A}), 9.2(\mathrm{~B})$ & 1.54 & 5 & 10 & 0.002 & 0.8 & 1 \\
\hline Ondansetron & 293 & 40 & 0 & 4 & 0.6 & $7.5(\mathrm{~B})$ & 1.76 & 4 & 0.01 & 1.6 & 4.5 & 2 \\
\hline Oseltamivir phosphate & 312 & 91 & 3 & 6 & 1.0 & $8.8(\mathrm{~B})$ & -0.19 & 75 & 521 & 0.000576 & 7 & 1 \\
\hline$\underline{\text { Oteracil potassium }}$ & 157 & 108 & 3 & 7 & -1.0 & $-3.1(A)$ & -5.54 & 24.5 & 7.42 & 0.0132 & 3 & 3 \\
\hline Paroxetine hydrochloride hydrate & 329 & 40 & 1 & 4 & 1.0 & $9.7(B)$ & 0.84 & 20 & 2 & 0.04 & 13.6 & 1 \\
\hline Pergolide mesylate & 314 & 44 & 1 & 2 & 0.8 & $8.0(B)$ & 2.39 & 0.25 & 5 & 0.0002 & 8.8 & 1 \\
\hline Perindopril erbumine & 368 & 96 & 2 & 7 & -1.0 & $3.2(A)$ & 0.07 & 4 & 500 & $3.2 \times 10^{-5}$ & 0.8 & 1 \\
\hline Pilsicainide hydrochloride hydrate & 272 & 32 & 1 & 3 & 1.0 & $10.4(B)$ & -0.12 & 50 & 100 & 0.002 & 4.4 & 1 \\
\hline Pioglitazone hydrochloride & 356 & 94 & 1 & 5 & -0.9 & $6.3(A)$ & 2.16 & 30 & 0.01 & 12 & 6.7 & 2 \\
\hline Pitavastatin calcium & 421 & 91 & 3 & 5 & -1.0 & $4.2(A)$ & 1.17 & 4 & 0.1 & 0.16 & 9.3 & 1 \\
\hline Polaprezinc & ND & ND & ND & ND & - & ND & ND & 75 & 0.01 & 30 & 2.2 & UC \\
\hline Pramipexole hydrochloride hydrate & 211 & 79 & 3 & 3 & 1.0 & $9.5(B)$ & -1.03 & 0.5 & 1000 & $2.0 \times 10^{-6}$ & 8.4 & 3 \\
\hline Pranlukast hydrate & 482 & 119 & 2 & 9 & -1.0 & $5.3(A)$ & 2.74 & 112.5 & 0.0012 & 375 & 1.2 & 2 \\
\hline Pravastatin sodium hydrate & 425 & 124 & 4 & 7 & -1.0 & $4.3(A)$ & 0.06 & 10 & 100 & 0.0004 & 2.7 & 1 \\
\hline Procaterol hydrochloride & 290 & 82 & 4 & 5 & 1.0 & $9.4(B)$ & -1.47 & 0.05 & 50 & $4.0 \times 10^{-6}$ & 3.8 & 3 \\
\hline Propiverine hydrochloride & 367 & 39 & 0 & 4 & 0.7 & $7.8(B)$ & 4.10 & 20 & 124 & 0.000645 & 10.7 & 1 \\
\hline Quetiapine fumarate & 384 & 74 & 1 & 5 & 0.2 & $6.7(B)$ & 1.92 & 200 & 3.38 & 0.2364 & 3.5 & 1 \\
\hline Ramosetron hydrochloride & 279 & 51 & 1 & 4 & 0.4 & $7.3(\mathrm{~B})$ & 1.80 & 0.005 & 575 & $3.48 \times 10^{-8}$ & 7 & 1 \\
\hline Ranitidine hydrochloride & 314 & 112 & 2 & 7 & 0.9 & $8.4(\mathrm{~B})$ & -1.47 & 150 & 1429 & 0.00042 & 2.7 & 3 \\
\hline Rebamipide & 371 & 96 & 3 & 6 & -1.0 & $3.4(\mathrm{~A})$ & -0.75 & 100 & 0.006 & 66.7 & 1.9 & 2 \\
\hline Risedronate sodium hydrate & 283 & 168 & 5 & 8 & -3.0 & $\begin{array}{c}1.4(A), 2.4(A) \\
6.0(A)\end{array}$ & -8.38 & 75 & 33 & 0.00909 & 1.5 & 3 \\
\hline Risperidone & 410 & 62 & 0 & 6 & 0.8 & $8.1(\mathrm{~B})$ & 0.99 & 2 & 0.01 & 0.8 & 0.3 & 1 \\
\hline
\end{tabular}


Table 1. (Continued)

\begin{tabular}{|c|c|c|c|c|c|c|c|c|c|c|c|c|}
\hline Drug & $\begin{array}{l}\text { MW } \\
\text { (free } \\
\text { form) }\end{array}$ & PSA $^{b}$ & $\mathrm{HBD}^{\mathrm{b}}$ & $\mathrm{HBA}^{\mathrm{b}}$ & $\begin{array}{c}\mathrm{NC}^{\mathrm{a}} \\
\mathrm{pH} 7.4\end{array}$ & $\mathrm{p} K_{\mathrm{a}}{ }^{\mathrm{b}}$ & $\log D_{6.5}^{b}$ & $\begin{array}{l}\text { Highest dose } \\
\text { strength } \\
(\mathrm{mg})^{c}\end{array}$ & $\begin{array}{l}\text { Solubility } \\
\text { in water } \\
(\mathrm{mg} / \mathrm{mL})^{c}\end{array}$ & Do & $\begin{array}{c}t_{1 / 2} \\
(\mathrm{~h})^{\mathrm{c}}\end{array}$ & $\begin{array}{l}\text { BCS } \\
\text { class }\end{array}$ \\
\hline Rizatriptan benzoate & 269 & 50 & 1 & 5 & 1.0 & $9.5(\mathrm{~B})$ & -0.70 & 10 & 34 & 0.00118 & 1.7 & 1 \\
\hline Sarpogrelate hydrochloride & 430 & 85 & 1 & 7 & -0.2 & $4.3(A), 8.1(B)$ & 1.37 & 100 & 1 & 0.4 & 0.8 & 1 \\
\hline Selegiline hydrochloride & 187 & 3 & 0 & 1 & 0.6 & 7.5 (B) & 1.81 & 2.5 & 1667 & $6.0 \times 10^{-6}$ & 5.3 & 1 \\
\hline Sertraline hydrochloride & 306 & 12 & 1 & 1 & 1.0 & $9.5(\mathrm{~B})$ & 2.45 & 100 & 2.70 & 0.148 & 25.1 & 1 \\
\hline Sildenafil citrate & 475 & 118 & 1 & 10 & 0.0 & - & 1.85 & 50 & 1 & 0.2 & 1.6 & 1 \\
\hline Simvastatin & 419 & 73 & 1 & 5 & 0.0 & - & 4.60 & 20 & 0.01 & 8 & 2.3 & 2 \\
\hline$\underline{\text { Solifenacin succinate }}$ & 362 & 171 & 5 & 12 & 1.0 & $9.0(B)$ & 1.36 & 5 & 610 & $3.28 \times 10^{-5}$ & 46.5 & 1 \\
\hline Sultamicillin tosilate & 595 & 216 & 3 & 13 & 0.2 & $6.8(\mathrm{~B})$ & -0.46 & 375 & 0.1 & 15 & 1.0 & 2 \\
\hline Tacrolimus hydrate & 804 & 178 & 3 & 13 & 0.0 & - & 4.10 & 5 & 0.01 & 2 & 32.0 & 2 \\
\hline Taltirelin hydrate & 405 & 171 & 5 & 12 & 0.2 & $6.7(B)$ & -2.73 & 5 & 208 & $9.6 \times 10^{-5}$ & 2 & 3 \\
\hline Tamoxifen citrate & 372 & 12 & 0 & 2 & 1.0 & $8.7(B)$ & 4.68 & 20 & 0.09 & 0.889 & 27.2 & 1 \\
\hline$\underline{\text { Tamsulosin hydrochloride }}$ & 409 & 108 & 3 & 7 & 1.0 & $8.8(\mathrm{~B})$ & -0.06 & 0.2 & 11.8 & $6.76 \times 10^{-5}$ & 11.7 & 1 \\
\hline$\underline{\text { Tegafur }}$ & 200 & 59 & 1 & 5 & -0.4 & $7.6(A)$ & -0.43 & 25 & 16.8 & 0.00595 & 1.9 & 1 \\
\hline Temocapril hydrochloride & 477 & 149 & 2 & 7 & -1.0 & $3.7(A)$ & 0.62 & 4 & 0.719 & 0.0223 & 0.2 & 1 \\
\hline Teprenone & 331 & 17 & 0 & 1 & 0.0 & - & 7.40 & 50 & 8.32 & 0.0240 & 1.8 & 1 \\
\hline Terbinafine hydrochloride & 291 & 3 & 0 & 1 & 0.2 & $6.9(\mathrm{~B})$ & 5.45 & 125 & 5.07 & 0.0987 & 6.2 & 1 \\
\hline Ticlopidine hydrochloride & 264 & 31 & 0 & 1 & 0.3 & $7.1(\mathrm{~B})$ & 3.41 & 100 & 58.8 & 0.0068 & 1.6 & 1 \\
\hline Tocopherol nicotinate & 536 & 48 & 0 & 4 & 0.0 & - & 11.14 & 200 & 0.01 & 80 & 4.3 & 2 \\
\hline Tramadol hydrochloride & 263 & 33 & 1 & 3 & 1.0 & $9.6(B)$ & -0.18 & 50 & 100 & 0.002 & 5.7 & 1 \\
\hline Tulobuterol hydrochloride & 228 & 32 & 2 & 2 & 1.0 & $9.6(B)$ & -0.17 & 1 & 714 & $5.6 \times 10^{-6}$ & 3.2 & 1 \\
\hline Ursodeoxycholic acid & 393 & 78 & 3 & 4 & -1.0 & $4.8(A)$ & 2.01 & 100 & 0.01 & 40 & 1.1 & 2 \\
\hline Valacyclovir hydrochloride & 324 & 147 & 5 & 10 & 0.7 & $7.8(\mathrm{~B})$ & -1.78 & 500 & 100 & 0.02 & 3.0 & 3 \\
\hline Valproate sodium & 144 & 37 & 1 & 2 & -1.0 & $4.8(\mathrm{~A})$ & 0.97 & 200 & 1000 & 0.0008 & 9.5 & 1 \\
\hline Valsartan & 436 & 112 & 2 & 8 & -2.0 & $3.6(A), 4.2(A)$ & -0.71 & 160 & 0.17 & 3.76 & 7.7 & 2 \\
\hline
\end{tabular}


Table 1. (Continued)

\begin{tabular}{|c|c|c|c|c|c|c|c|c|c|c|c|c|}
\hline Drug & $\begin{array}{l}\text { MW } \\
\text { (free } \\
\text { form) }\end{array}$ & PSA $^{b}$ & $H_{B D}^{b}$ & $\mathrm{HBA}^{b}$ & $\begin{array}{c}\mathrm{NC}^{\mathrm{a}} \\
\mathrm{pH} 7.4\end{array}$ & $\mathrm{p} K_{\mathrm{a}}^{\mathrm{b}}$ & $\log D_{6.5}^{b}$ & $\begin{array}{l}\text { Highest dose } \\
\text { strength } \\
(\mathrm{mg})^{\mathrm{c}}\end{array}$ & $\begin{array}{l}\text { Solubility } \\
\text { in water } \\
(\mathrm{mg} / \mathrm{mL})^{c}\end{array}$ & Do & $\begin{array}{c}t_{1 / 2} \\
\text { (h) }\end{array}$ & $\begin{array}{l}\text { BCS } \\
\text { class }\end{array}$ \\
\hline Voglibose & 267 & 154 & 8 & 8 & 0.2 & $6.8(B)$ & -3.57 & 0.3 & 1000 & $1.2 \times 10^{-6}$ & 5.3 & 3 \\
\hline Zolmitriptan & 287 & 57 & 2 & 5 & 1.0 & $9.5(B)$ & -0.29 & 2.5 & 0.1 & 0.1 & 2.9 & 1 \\
\hline Zolpidem tartrate & 307 & 38 & 0 & 4 & 0.2 & $6.8(B)$ & 2.78 & 10 & 8.9 & 0.00449 & 1.9 & 1 \\
\hline Zonizamide & 212 & 95 & 2 & 5 & 0.0 & - & 0.45 & 25 & 0.270 & 0.37 & 119.1 & 1 \\
\hline
\end{tabular}

$\mathrm{ND}=$ no data.

$\mathrm{UC}=$ unclassifiable.

${ }^{\text {a }}$ Net charge at pH 7.4 calculated by Eq. 1.

${ }^{\mathrm{b}}$ Calculated value (ACD/Labs Software V 14.0.0)

${ }^{\mathrm{C}}$ Data from prescription information (Supplement Tables 1 and 2) otherwise noted.

d Underscored drugs correspond to ODTs.

${ }^{\mathrm{e}}$ Drugs in italics correspond to IR formulations.

${ }^{\mathrm{f}}$ Reference [12] 
Table 2. Minimum, maximum, and median of biopharmaceutical properties of ODT and IR formulation drugs.

\begin{tabular}{|c|c|c|c|}
\hline \multicolumn{2}{|c|}{ Biopharmaceutical properties } & \multirow{2}{*}{$\begin{array}{c}\text { ODT } \\
56\end{array}$} & \multirow{2}{*}{$\frac{\text { IR formulation }}{113}$} \\
\hline \multirow{3}{*}{$M W$ of free form } & $n$ & & \\
\hline & Median & 359 & 371 \\
\hline & Min. - Max. & $146-1069$ & $136-1344$ \\
\hline \multirow{3}{*}{ PSA } & $\mathrm{n}$ & 56 & 111 \\
\hline & Median & 70 & 84 \\
\hline & Min. - Max. & $3-486$ & $3-321$ \\
\hline \multirow{3}{*}{ HBD } & $\mathrm{n}$ & 56 & 111 \\
\hline & Median & 1 & 2 \\
\hline & Min. - Max. & $0-18$ & $0-14$ \\
\hline \multirow{3}{*}{ HBA } & $\mathrm{n}$ & 56 & 111 \\
\hline & Median & 5 & 6 \\
\hline & Min. - Max. & $1-26$ & $1-23$ \\
\hline \multirow{3}{*}{$\mathrm{NC}^{\mathrm{a}} \mathrm{pH} 7.4$} & $\mathrm{n}$ & 56 & 111 \\
\hline & Median & 0.2 & 0.0 \\
\hline & Min. - Max. & $-2.0-1.0$ & $-3.0-1.8$ \\
\hline \multirow{3}{*}{$\log D_{6.5}$} & $\mathrm{n}$ & 56 & 111 \\
\hline & Median & 1.02 & 0.97 \\
\hline & Min. - Max. & $-7.34-5.49$ & $-8.44-11.14$ \\
\hline \multirow{3}{*}{$\begin{array}{l}\text { The highest dose } \\
\text { strength } \\
\text { (mg) }\end{array}$} & $\mathrm{n}$ & 57 & 113 \\
\hline & Median & 10 & 30 \\
\hline & Min. - Max. & $0.005-200$ & $0.003-900$ \\
\hline \multirow{3}{*}{$\begin{array}{c}\text { Solubility in water } \\
(\mathrm{mg} / \mathrm{mL})\end{array}$} & $\mathrm{n}$ & 57 & 113 \\
\hline & Median & 1.43 & 3.33 \\
\hline & Min. - Max. & $0.003-1667$ & $0.0012-1429$ \\
\hline \multirow{3}{*}{ Do } & $n$ & 57 & 113 \\
\hline & Median & 0.1 & 0.04 \\
\hline & Min. - Max. & $3.48 \times 10^{-8}-66.7$ & $5.4 \times 10^{-8}-375$ \\
\hline \multirow{3}{*}{$\begin{array}{l}\text { Elimination } t_{1 / 2} \\
\text { (h) }\end{array}$} & $\mathrm{n}$ & 57 & 113 \\
\hline & Median & 6.7 & 3.3 \\
\hline & Min. - Max. & $0.9-128$ & $0.2-70.7$ \\
\hline
\end{tabular}

${ }^{\mathrm{a}}$ Net charge at $\mathrm{pH} 7.4$ calculated by Eq. 1.

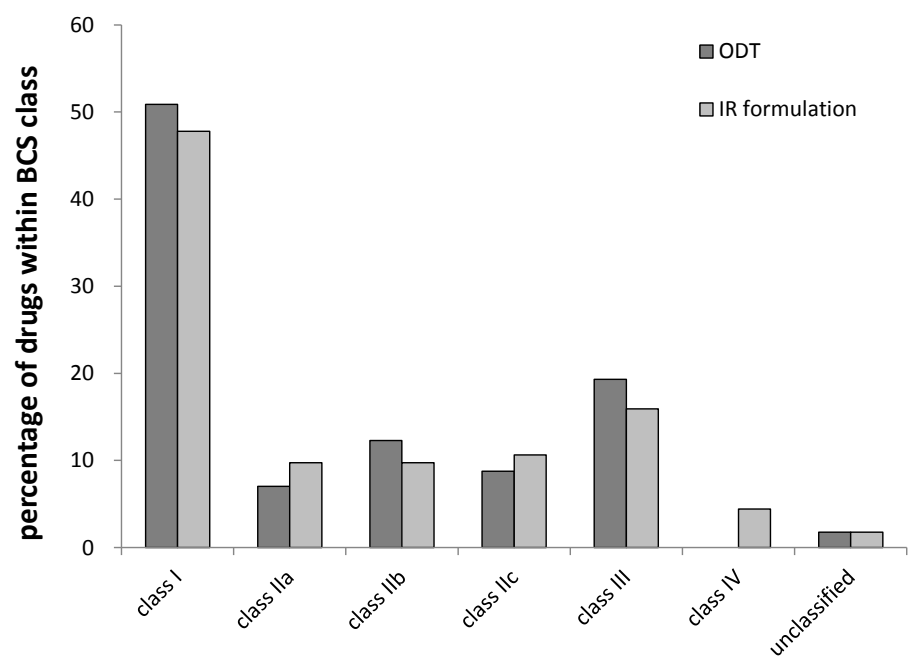

Figure 2. Provisional BCS classification of drugs in ODTs and IR formulations. 


\section{Discussion}

A significant difference was seen in the highest dose strength between the ODT and IR formulation. The ODT would have to have a feasible tablet size and drug loading $[13,14]$. Therefore, a drug with a high dose strength (>200 $\mathrm{mg}$ ) would be less suitable an ODT.

There was no significant difference in the solubility in water and Do between the ODT and IR formulation. This result suggests that many drugs can be developed as an ODT regardless of their solubility and Do (e.g., bicalutamide: $S=0.01 \mathrm{mg} / \mathrm{mL}$, Do $=32$, cilostazole: $S=0.01 \mathrm{mg} / \mathrm{mL}$, Do $=40$ ). Furthermore, an ODT shows BE with a corresponding IR formulation with and without water intake. Therefore, even though it is counterintuitive, water intake may have little effect on the dissolution and oral absorption of low solubility drugs. Previously, Sumesen et al. reported that the oral absorption of danazol was not significantly altered when administered together with $1000 \mathrm{~mL}$ of water compared to when administered with $200 \mathrm{~mL}$ [15]. Danazol, which has poor water solubility $(0.2 \mu \mathrm{g} / \mathrm{mL})$ and high permeability $\left(\log D_{6.5}=\right.$ 4.5), is a typical BCS class II drug [16].

The drugs that have been developed as ODTs tended to have a longer elimination $t_{1 / 2}$. Previously, we reported that the elimination $t_{1 / 2}$ of drugs influence the $\mathrm{BE}$ of $C_{\max }$ [17]. For the drugs with high permeability and short elimination $t_{1 / 2}, \mathrm{BE}$ of $\mathrm{Cmax}$ between two formulations with different dissolution rates would become more difficult to prove. This point has been suggested by several articles [18-21]. A drug with a long elimination $t_{1 / 2}$ might be suitable for an ODT.

The proportion of bases was larger in the ODT than in the IR formulation. It is well known that the basic lipophilic drugs have a large distribution volume, and a long elimination $t_{1 / 2}$ due to wide tissue distribution [22]. A significant difference was seen in elimination $t_{1 / 2}$ between acids, bases, and undissociated drugs in the IR formulation ( $p=0.04$ ) (Figure 3 ).

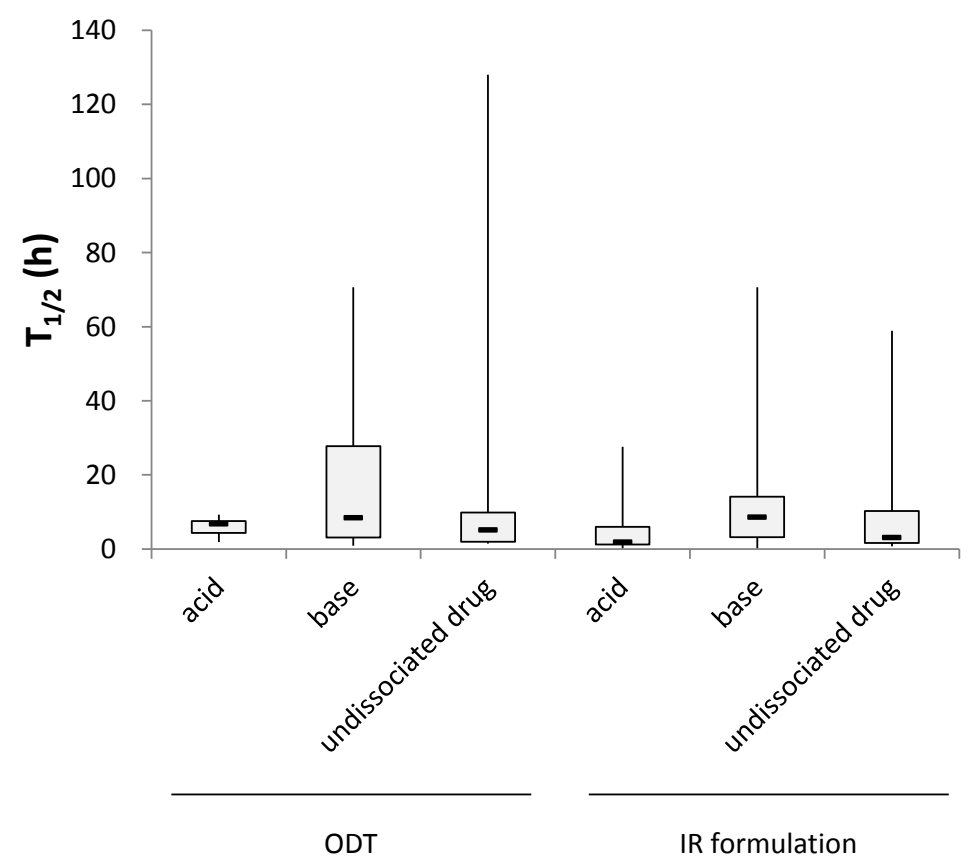

Figure 3. Box and whisker plot of elimination $t_{1 / 2}$ of acid, base, and undissociated drugs

The biowaiver schemes (BWS) has been discussed based on BCS proposed by Amidon et al. in 1995 [5]. In 2000, the US Food and Drug Administration (FDA) adopted the BCS-BWS [23]. The guideline allows BCS class I drugs which show rapid dissolution ( $>85 \%$ dissolution in $30 \mathrm{~min}$ ) to waive clinical BE studies. The World Health Organization (WHO) and other regulatory agencies followed the BCS-BWS [24-27]. However, 
there are some differences among these guidelines, e.g. solubility $\mathrm{pH}$ range, criteria for high permeability, and definition of the dose used for the Do calculation [28]. One of the most significant differences is about the biowaiver for BCS class III drugs. WHO, European Union, and Canada accept biowaiver for BCS class III drugs which show very rapid dissolution (>85\% dissolution in $15 \mathrm{~min}$ ), while only BCS class I drugs are eligible for biowaiver in the US FDA and Korea FDA guideline. However, there are several computer simulation and experimental studies on the biowaiver for BCS class III drugs, suggesting that BCS class III drugs are suitable for biowaiver [20,21,29-33]. In addition, it has been pointed out that many BCS class III drugs show $B E$ even when the dissolution profiles are different between the test and reference drugs, for example famotidine, hydrochlorothiazide, and cimetidine [33,34]. Moreover, WHO adopts the possibility of biowaiver for BCS class Ila drugs with low solubility at acidic $\mathrm{pH}$ and high solubility at neutral $\mathrm{pH}$ that are absorbed completely. However, it was reported that, in the case of ibuprofen, the typically BCS class Ila drug, the $\mathrm{BE}$ of Cmax is more sensitive to the difference of dissolution rates [35,36].

The BCS class distribution of ODTs and IR formulations may reflect their non-BE risk due to the difference of the dissolution rates. Based on the BCS-BWS, BE is most easily established for BCS class I drugs. However, no difference in the distribution of the BCS classes, including subclasses, between the ODTs and IR formulations was observed in this study. This result may suggest that a BCS class I drug would not necessarily be suitable to show BE. Ramirez et al. reported that in the 124 clinical BE studies there is no difference in the number of subjects in the BE study and the inter- and intra-subject variability for $C_{\max }$ or AUC between four BCS classes [37]. All the BCS classes drugs have the risk of non-BE. Their results also showed that all of the bioinequivalent parameters in BCS class I drugs was $C_{\max }$, but not AUC. The results of the present study suggest that the elimination $t_{1 / 2}$ would affect the success rate of a $\mathrm{BE}$ study more significantly than the BCS class of a drug (e.g. ibuprofen).

In conclusion, drugs with a lower dose strength $(<10 \mathrm{mg})$ and a longer elimination $t_{1 / 2}(>6.5 \mathrm{~h})$ were suggested to be more suitable for an ODT. However, the distributions of the ODT and IR formulation among each BCS class were similar, suggesting that the BCS classes are irrelevant to the development risk of ODTs.

\section{References}

[1] S. V. Sastry, J. R. Nyshadham, J. A. Fix. Recent technological advances in oral drug delivery - a review. Pharm. Sci. Technolo. Today 3 (2000) 138-145.

[2] D. Hobbs, J. Karagianis, T. Treuer, J. Raskin. An in vitro analysis of disintegration times of different formulations of olanzapine orodispersible tablet: a preliminary report. Drugs $R D 13$ (2013) 281-288.

[3] T. Takagi, C. Ramachandran, M. Bermejo, S. Yamashita, L. X. Yu, G. L. Amidon. A provisional biopharmaceutical classification of the top 200 oral drug products in the United States, Great Britain, Spain, and Japan. Mol. Pharm. 3 (2006) 631-643.

[4] C. A. Lipinski, F. Lombardo, B. W. Dominy, P. J. Feeney. Experimental and computational approaches to estimate solubility and permeability in drug discovery and development settings. Advanced Drug Delivery Reviews 64 (2012) 4-17.

[5] G. Amidon, H. Lennernäs, V. Shah, J. Crison. A theoretical basis for a biopharmaceutic drug classification: the correlation of in vitro drug product dissolution and in vivo bioavailability. Pharm. Res. 12 (1995) 413-420.

[6] S. K. Poole, S. Patel, K. Dehring, H. Workman, C. F. Poole. Determination of acid dissociation constants by capillary electrophoresis. J. Chromatogr. A 1037 (2004) 445-454.

[7] Y. Tsume, D. M. Mudie, P. Langguth, G. E. Amidon, G. L. Amidon. The Biopharmaceutics Classification System: subclasses for in vivo predictive dissolution (IPD) methodology and IVIVC. Eur. J. Pharm. Sci. 57 (2014) 152-163. 
[8] N. A. Kasim, M. Whitehouse, C. Ramachandran, M. Bermejo, H. Lennernäs, A. S. Hussain, H. E. Junginger, S. A. Stavchansky, K. K. Midha, V. P. Shah, G. L. Amidon. Molecular properties of WHO essential drugs and provisional biopharmaceutical classification. Mol. Pharm. 1 (2003) 85-96.

[9] H. Lennernäs. Intestinal permeability and its relevance for absorption and elimination. Xenobiotica 37 (2007) 1015-1051.

[10] A. Avdeef. Absorption and Drug Development, Second Edition. John Wiley \& Sons, Inc (2012)

[11] K. Sugano. Biopharmaceutics Modeling and Simulations. Wiley (2012)

[12] B. Reigner, J. Verweij, L. Dirix, J. Cassidy, C. Twelves, D. Allman, E. Weidekamm, B. Roos, L. Banken, M. Utoh. Effect of food on the pharmacokinetics of capecitabine and its metabolites following oral administration in cancer patients. Clin. Cancer Res. 4 (1998) 941-948.

[13] J. J. Hirani, D. A. Rathod, K. R. Vadalia. Orally disintegrating tablets: a review. Trop. J. Pharm. Res. 8 (2009) 161-172.

[14] T. K. Ghosh, W. R. Pfiste. Drug delivery to the oral cavity; chapter 14. Quick-dissolving oral dosage forms Taylor \& Francis Group, LLC (2005) 337-356.

[15] V. H. Sunesen, R. Vedelsdal, H. G. Kristensen, L. Christrup, A. Mullertz. Effect of liquid volume and food intake on the absolute bioavailability of danazol, a poorly soluble drug. Eur. J. Pharm. Sci. 24 (2005) 297-303.

[16] K. Sugano. Fraction of a dose absorbed estimation for structurally diverse low solubility compounds. Int. J. Pharm. 405 (2011) 79-89.

[17] K. Sugano, K. Terada. Rate- and Extent-Limiting Factors of Oral Drug Absorption: Theory and Applications. J. Pharm. Sci. 104 (2015) 2777-2788.

[18] S. Colon-Useche, I. Gonzalez-Alvarez, V. Mangas-Sanjuan, M. Gonzalez-Alvarez, P. Pastoriza, I. Molina-Martinez, M. Bermejo, A. Garcia-Arieta. Investigating the Discriminatory Power of BCSBiowaiver in Vitro Methodology to Detect Bioavailability Differences between Immediate Release Products Containing a Class I Drug. Mol. Pharm. 12 (2015) 3167-3174.

[19] A. Garcia-Arieta, J. Gordon, L. Gwaza, V. Mangas-Sanjuan, C. Alvarez, J. J. Torrado. Agitation rate and time for complete dissolution in BCS biowaivers based on investigation of a BCS biowaiver for dexketoprofen tablets. Mol. Pharm. 12 (2015) 3194-3201.

[20] U. Fagerholm. Evaluation and suggested improvements of the Biopharmaceutics Classification System (BCS). J. Pharm. Pharmacol. 59 (2007) 751-757.

[21] H. Kortejarvi, A. Urtti, M. Yliperttula. Pharmacokinetic simulation of biowaiver criteria: the effects of gastric emptying, dissolution, absorption and elimination rates. Eur. J. Pharm. Sci. 30 (2007) 155-166.

[22] G. A. Siebert, D. Y. Hung, P. Chang, M. S. Roberts. Ion-trapping, microsomal binding, and unbound drug distribution in the hepatic retention of basic drugs. J. Pharmacol. Exp. Ther. 308 (2004) 228-235.

[23] FDA. Waiver of in vivo bioavailability and bioequivalence studies for immediate-release solid oral dosage forms based on a biopharmaceutics classification system. FDA Guidance for Industry (2000)

[24] WHO. Proposal to waive in vivo bioequivalence requirements for WHO Model List of Essential Medicines immediate-release, solid oral dosage forms. WHO Technical Report Series, No. 937, Annex 8 (2006)

[25] EMA. Guideline on the investigation of bioequivalence. CPMP/EWP/QWP/1401/98 (2010)

[26] H. Canada. Biopharmaceutics classification system based biowaiver. Guidance document (2013)

[27] K. FDA. Bioequivalence study. Guidance document (2008)

[28] B. Davit, A. C. Braddy, D. P. Conner, L. X. Yu. International guidelines for bioequivalence of systemically available orally administered generic drug products: a survey of similarities and differences. AAPS J 15 (2013) 974-990.

[29] L. Yu, G. Amidon, J. Polli, H. Zhao, M. Mehta, D. Conner, V. Shah, L. Lesko, M.-L. Chen, V. L. Lee, A. Hussain. Biopharmaceutics Classification System: The Scientific Basis for Biowaiver Extensions. Pharm. Res. 19 (2002) 921-925. 
[30] C. L. Cheng, L. X. Yu, H. L. Lee, C. Y. Yang, C. S. Lue, C. H. Chou. Biowaiver extension potential to BCS Class III high solubility-low permeability drugs: bridging evidence for metformin immediate-release tablet. Eur. J. Pharm. Sci. 22 (2004) 297-304.

[31] E. Jantratid, S. Prakongpan, G. L. Amidon, J. B. Dressman. Feasibility of biowaiver extension to biopharmaceutics classification system class III drug products. Clin. Pharmacokinet. 45 (2006) 385399.

[32] Y. Tsume, G. L. Amidon. The biowaiver extension for BCS class III drugs: the effect of dissolution rate on the bioequivalence of BCS class III immediate-release drugs predicted by computer simulation. Mol. Pharm. 7 (2010) 1235-1243.

[33] A. Ono, K. Sugano. Application of the BCS biowaiver approach to assessing bioequivalence of orally disintegrating tablets with immediate release formulations. Eur. J. Pharm. Sci. 64 (2014) 37-43.

[34] E. Jantratid, S. Prakongpan, J. B. Dressman, G. L. Amidon, H. E. Junginger, K. K. Midha, D. M. Barends. Biowaiver monographs for immediate release solid oral dosage forms: cimetidine. J. Pharm. Sci. 95 (2006) 974-984.

[35] C. Alvarez, I. Nunez, J. J. Torrado, J. Gordon, H. Potthast, A. Garcia-Arieta. Investigation on the possibility of biowaivers for ibuprofen. J. Pharm. Sci. 100 (2011) 2343-2349.

[36] A. Garcia-Arieta, J. Gordon, H. Potthast. On the Biopharmaceutics Classification System Biowaiver of Ibuprofen. J. Pharm. Sci. 104 (2015) 2429-2432.

[37] E. Ramirez, O. Laosa, P. Guerra, B. Duque, B. Mosquera, A. M. Borobia, S. H. Lei, A. J. Carcas, J. Frias. Acceptability and characteristics of 124 human bioequivalence studies with active substances classified according to the Biopharmaceutic Classification System. Br. J. Clin. Pharmacol. 70 (2010) 694-702. 
Supplement Table 1. ODT drug list used in this study

Drug
Acarbose
Ambroxol hydrochloride
Amlodipine besylate
Aripiprazole
Bepotastine besylate
Bicalutamide
Brotizolam
Cetirizine hydrochloride
Cilostazol
Desmopressin acetate hydrate
Dienogest
Domperidone
Donepezil hydrochloride
Doxazosin mesylate
Droxidopa
Ebastine
Famotidine
Fexofenadine hydrochloride
Galantamine hydrobromide
Gimeracil
Glimepiride
Hydrochlorothiazide
Imidafenacin
Irsogladine maleate

\section{Prescription information}

http://www.info.pmda.go.jp/go/interview/1/6300043969003F3037 $10051 \mathrm{~F}$

http://www.info.pmda.go.jp/go/interview/1/300119 2239001G1039 $1061 \quad 1 \mathrm{~F}$

http://www.info.pmda.go.jp/go/interview/2/671450 2171022F1029 $2 \quad 1 \mathrm{~F}$

http://www.info.pmda.go.jp/go/interview/3/400093 2171022F1045 $3017 \quad 1 \mathrm{~F}$

http://www.info.pmda.go.jp/go/interview/1/180078 1179045F4022 $1012 \quad 1 \mathrm{~F}$

http://www.info.pmda.go.jp/go/interview/1/400315 4490022F3022 $1090 \quad 1 \mathrm{~F}$

http://www.info.pmda.go.jp/go/interview/1/670227 4291009F1039 $1171 \quad 1 \mathrm{~F}$

http://www.info.pmda.go.jp/go/interview/1/650168 1124009F2025 $114 \mathrm{X} 1 \mathrm{~F}$

http://www.info.pmda.go.jp/go/interview/1/300119 4490020F1225 1051 1F

http://www.info.pmda.go.jp/go/interview/1/180078 3399002F3020 1005 1F

http://www.info.pmda.go.jp/go/interview/1/670666 2419001F1023 2 1F

http://www.info.pmda.go.jp/go/interview/1/790005 2499010F1023 1 M02 1F http://www.info.pmda.go.jp/go/interview/1/230124 2399005F3020 $1004 \quad 1 F$ http://www.info.pmda.go.jp/go/interview/1/170033 1190012F3029 $1028 \quad 1 \mathrm{~F}$ http://www.info.pmda.go.jp/go/interview/2/671450 2149026F1026 2 1F

http://www.info.pmda.go.jp/go/interview/2/400093 1169006C1039 2009 1F http://www.info.pmda.go.jp/go/interview/1/400093 4490019F1028 1018 1F http://www.info.pmda.go.jp/go/interview/1/800126 2325003F1024 1 1F http://www.info.pmda.go.jp/go/interview/1/780069 4490023F1024 1021 1F http://www.info.pmda.go.jp/go/interview/1/800155 1190019F1028 1005 1F http://www.info.pmda.go.jp/go/interview/1/400107 4229101F1026 1 10E $1 \mathrm{~F}$ http://www.info.pmda.go.jp/go/interview/1/780069 3961008F4070 $10201 \mathrm{~F}$ http://www.info.pmda.go.jp/go/interview/1/480235 2132004F1103 $1002 \quad 1 F$ http://www.info.pmda.go.jp/go/interview/1/230109 2590013F1027 1 a13 $1 \mathrm{~F}$ http://www.info.pmda.go.jp/go/interview/1/180188 2590013F1035 $10091 \mathrm{~F}$ http://www.info.pmda.go.jp/go/interview/1/530263 2329020F3020 1 05F $1 \mathrm{~F}$ 
Lafutidine

Lansoprazole

Loratadine

Memantine hydrochloride

Midodrine hydrochloride

Miglitol

Naftopizil

Olanzapine

Olopatadine hydrochloride

Ondansetron hydrochloride

Oteracil potassium

Paroxetine hydrochloride hydrate

Pioglitazone hydrochloride

Pitavastatin calcium

Polaprezinc

Pramipexole hydrochloride hydrate

Ramosetron hydrochloride

\author{
Rebamipide \\ Risperidone \\ Rizatriptan benzoate \\ Selegiline hydrochloride \\ Sertraline hydrochloride \\ Sildenafil citrate \\ Solifenacin succinate \\ Taltirelin hydrate \\ Tamsulosin hydrochloride \\ Tegafur
}

http://www.info.pmda.go.jp/go/interview/1/400107 2325006F3020 1 04E 1F http://www.info.pmda.go.jp/go/interview/1/400256 2329023F1020 1006 1F http://www.info.pmda.go.jp/go/interview/1/170050 4490027F1022 $1015 \quad 1 F$ http://www.info.pmda.go.jp/go/interview/1/430574 1190018F1023 1 M09 1F http://www.info.pmda.go.jp/go/interview/1/400059 2160002F2024 $10041 \mathrm{~F}$ http://www.info.pmda.go.jp/go/interview/1/300297 39690A0F1026 $10011 \mathrm{~F}$ http://www.info.pmda.go.jp/go/interview/1/100898 2590009F4020 11 1F http://www.info.pmda.go.jp/go/interview/1/530471 1179044F4028 $118 \mathrm{~F} \quad 1 \mathrm{~F}$ http://www.info.pmda.go.jp/go/interview/1/230124 4490025F3026 $1006 \quad 1 \mathrm{~F}$ http://www.info.pmda.go.jp/go/interview/1/340278 2391006F1023 11 1F http://www.info.pmda.go.jp/go/interview/1/400107 4229101F1026 1 10E $1 \mathrm{~F}$ http://www.info.pmda.go.jp/go/interview/1/480235 1179041F1254 1005 1F http://www.info.pmda.go.jp/go/interview/1/400256 3969007F3027 $1007 \quad 1 \mathrm{~F}$ http://www.info.pmda.go.jp/go/interview/1/270072 2189016F4027 $1005 \quad 1 F$ http://www.pmda.go.jp/PmdaSearch/iyakuDetail/ResultDataSetPDF/380077 2329027F1029 104 http://www.info.pmda.go.jp/go/interview/1/480235 1169012F3025 $1004 \quad 1 F$ http://www.info.pmda.go.jp/go/interview/1/800126 2399014F3029 1 1F http://www.info.pmda.go.jp/go/interview/1/800126 2391004F1024 1 1F http://www.info.pmda.go.jp/go/interview/1/530113 2329021F2028 $1002 \quad 1 F$ http://www.info.pmda.go.jp/go/interview/1/800155 1179038F5029 1005 1F http://www.info.pmda.go.jp/go/interview/2/230109 2160006F1026 2011 1F http://www.info.pmda.go.jp/go/interview/1/170654 1169010F2020 1 S03 1F http://www.info.pmda.go.jp/go/interview/2/671450 1179046F1028 $2 \quad 1 \mathrm{~F}$ http://www.info.pmda.go.jp/go/interview/1/480235 259000AF3027 $10021 \mathrm{~F}$ http://www.info.pmda.go.jp/go/interview/1/800126 2590011F3020 1 1F http://www.info.pmda.go.jp/go/interview/1/400315 1190014F2021 1050 1F http://www.info.pmda.go.jp/go/interview/1/800126 2590008F1026 1 1F http://www.info.pmda.go.jp/go/interview/1/400107 4229101F1026 1 10E 1F 
Tramadol hydrochloride

Valsartan

Voglibose

Zolmitriptan

Zolpidem tartrate

Zonizamide http://www.info.pmda.go.jp/go/interview/1/530263 1149038F1024 1 01F 1F

http://www.info.pmda.go.jp/go/interview/1/300242 2149041F5026 5 DIO $1 F$

http://www.info.pmda.go.jp/go/interview/1/400256 3969004F3023 $1006 \quad 1 F$

http://www.info.pmda.go.jp/go/interview/1/670227 2160004F2023 1101 1F

http://www.info.pmda.go.jp/go/interview/3/800126 1129009F1025 3 1F

http://www.info.pmda.go.jp/go/interview/1/400093 1169015F20221004 1F 
Supplement Table 2. IR formulation drug list used in this study

\section{Drug}

\section{Acarbose}

Acetaminophen

Acetylsalicylic acid

Acyclovir

Alendronate sodium hydrate

Alfacalcidol

Allopurinol

Ambroxol hydrochloride

Amlodipine besylate

Atenolol

Atorvastatin calcium

Azithromycin hydrate

Azulene sulfonate

Benidipine hydrochloride

Beraprost sodium

Bicalutamide

Bisoprolol fumarate

Brotizolam

Cabergoline

Camostat mesylate

Candesartan cilexetil

Carvedilol

Cefcapene pivoxil hydrochloride

Cefdinir

Cefditoren pivoxil

Cefotiam hexetil hydrochloride

\section{Prescription information}

http://www.info.pmda.go.jp/go/interview/1/6300043969003F3037 1005 1F http://www.info.pmda.go.jp/go/interview/3/671610 1141007F1063 3 15L 1F http://www.info.pmda.go.jp/go/interview/1/630004 3399007H1021 $1002 \quad 1 F$ http://www.info.pmda.go.jp/go/interview/W/340278 6250002F1025 1001 1F http://www.info.pmda.go.jp/go/interview/2/170050 3999018F1021 2018 1F http://www.info.pmda.go.jp/go/interview/1/450045 3112001M1046 $1007 \quad 1 F$ http://www.info.pmda.go.jp/go/interview/W/340278 3943001F1314 $11 \mathrm{~F}$ http://www.info.pmda.go.jp/go/interview/1/650168 2239001N1135 $1148 \quad 1 \mathrm{~F}$ http://www.info.pmda.go.jp/go/interview/2/671450 2171022F1029 $2 \quad 1 \mathrm{~F}$ http://www.info.pmda.go.jp/go/interview/3/670227 2123011F1155 11131 1F http://www.info.pmda.go.jp/go/interview/1/800126 2189015F1023 $11 \mathrm{~F}$ http://www.info.pmda.go.jp/go/interview/2/671450 6149004F1028 2 1F http://www.info.pmda.go.jp/go/interview/1/530263 2323001F1225 1 04F $1 \mathrm{~F}$ http://www.info.pmda.go.jp/go/interview/1/230124 2171021F1024 $1001 \quad 1 F$ http://www.info.pmda.go.jp/go/interview/1/480220 3399005F1021 1 A06 1F http://www.info.pmda.go.jp/go/interview/1/670227 4291009F1039 $1171 \quad 1 \mathrm{~F}$ http://www.info.pmda.go.jp/go/interview/1/400315 2123016F1107 $11201 \mathrm{~F}$ http://www.info.pmda.go.jp/go/interview/1/650168 1124009F2025 $114 \mathrm{X} 1 \mathrm{~F}$ http://www.info.pmda.go.jp/go/interview/4/671450 1169011F1028 4 1F http://www.info.pmda.go.jp/go/interview/1/180188 3999003F1297 1005 1F http://www.info.pmda.go.jp/go/interview/1/400256 2149040F1026 $10041 \mathrm{~F}$ http://www.info.pmda.go.jp/go/interview/2/430574 2149032F1021 2 A13 1F http://www.info.pmda.go.jp/go/interview/1/340018 6132016F1023 1011 1F http://www.info.pmda.go.jp/go/interview/3/800126 6132013M1029 3 1F http://www.info.pmda.go.jp/go/interview/1/780009 6132015F1037 1 01A 1F http://www.pmda.go.jp/PmdaSearch/iyakuDetail/ResultDataSetPDF/400256 6132012F1025 112 
Cetirizine hydrochloride

\section{Cilostazol}

Clarithromycin

Cyclosporin a

Diclofenac sodium

Donepezil hydrochloride

Doxazosin mesylate

Doxifluridine

Ebastine

Enalapril maleate

Epalrestat

Eperisone hydrochloride

Epinastine hydrochloride

Ethyl icosapentate

Etizolam

Famotidine

Fexofenadine hydrochloride

Fluconazole

Flurbiprofen

Fluvastatin sodium

Fluvoxamine maleate

Fursultiamine

Gefitinib

Glimepiride

Granisetron hydrochloride

Imatinib mesylate

Imidapril hydrochloride

Itraconazole http://www.info.pmda.go.jp/go/interview/2/820110 4490020F1020 $2013 \quad 1 \mathrm{~F}$ http://www.info.pmda.go.jp/go/interview/1/180078 3399002F3020 $1005 \quad 1 F$ http://www.info.pmda.go.jp/go/interview/1/400059 6149003F2038 $1028 \quad 1 \mathrm{~F}$ http://www.info.pmda.go.jp/go/interview/2/300242 3999004M3021 2 NEO 1F http://www.info.pmda.go.jp/go/interview/1/300242 1147002F1560 1 VOL 1F http://www.info.pmda.go.jp/go/interview/1/170033 1190012F3029 $1028 \quad 1 \mathrm{~F}$ http://www.info.pmda.go.jp/go/interview/2/671450 2149026F1026 2 1F

http://www.info.pmda.go.jp/go/interview/2/450045 4223004M1027 $20041 \mathrm{~F}$ http://www.info.pmda.go.jp/go/interview/1/400093 4490019F1028 $1018 \quad 1 \mathrm{~F}$ http://www.info.pmda.go.jp/go/interview/2/170050 2144002F1024 $2016 \quad 1 F$ http://www.info.pmda.go.jp/go/interview/1/180188 3999013F1231 1004 1F http://www.info.pmda.go.jp/go/interview/1/170033 1249009D1030 $1007 \quad 1 \mathrm{~F}$ http://www.info.pmda.go.jp/go/interview/1/650168 4490014F1025 1119 1F http://www.info.pmda.go.jp/go/interview/1/790005 3399004M2022 1 M03 1F http://www.info.pmda.go.jp/go/interview/2/400315 1179025C1054 $2 \quad 1501 \mathrm{~F}$ http://www.info.pmda.go.jp/go/interview/1/800126 2325003F1024 11 1F http://www.info.pmda.go.jp/go/interview/1/780069 4490023F1024 1021 1F http://www.info.pmda.go.jp/go/interview/2/671450 6290002M1020 2 1F http://www.info.pmda.go.jp/go/interview/1/200022 1149011D1032 $10801 \mathrm{~F}$ http://www.info.pmda.go.jp/go/interview/3/300242 2189012F1020 3 LOC 1F http://www.info.pmda.go.jp/go/interview/1/780009 1179039F1028 $11 \mathrm{~F}$ http://www.pmda.go.jp/PmdaSearch/iyakuDetail/ResultDataSetPDF/400256 3122007F2039 110 http://www.info.pmda.go.jp/go/interview/1/6702274291013F102711 192 1F http://www.info.pmda.go.jp/go/interview/1/780069 3961008F4070 $10201 \mathrm{~F}$ http://www.info.pmda.go.jp/go/interview/1/450045 2391002C1037 $10101 \mathrm{~F}$ http://www.info.pmda.go.jp/go/interview/1/300242 4291011F1028 1 GLI 1F http://www.info.pmda.go.jp/go/interview/1/400315 2144008F1021 $1090 \quad 1 F$ http://www.info.pmda.go.jp/go/interview/1/800155 6290004M1029 2008 1F 
Ketoprofen $^{\text {a }}$

Ketotifen fumarate

L-carbocysteine

Levofloxacin

Limaprost alfadex

Loratadine

Losartan potassium

Loxoprofen sodium hydrate

Manidipine hydrochloride

Mecobalamin

Meloxicam

Menatetrenone

Mesalazine

Methylmethionine sulfonium

chloride

Mexiletine hydrochloride

Montelukast sodium

Mosapride citrate hydrate

Nicardipine hydrochloride

Nicergoline

Nicorandil

Nifedipine

Nilvadipine

Nizatidine

Olanzapine

Olopatadine hydrochloride

Oseltamivir phosphate

Paroxetine hydrochloride hydrate http://www.info.pmda.go.jp/go/interview/2/650208 1149700J1035 2 1F

http://www.info.pmda.go.jp/go/interview/1/300242 4490003M1263 1 Z-C 1F

http://www.info.pmda.go.jp/go/interview/1/230109 2233002F1174 $1001 \quad 1 F$

http://www.info.pmda.go.jp/go/interview/1/430574 6241013C2024 1 c11 $1 \mathrm{~F}$

http://www.info.pmda.go.jp/go/interview/1/180188 3399003F1073 $1010 \quad 1 F$

http://www.info.pmda.go.jp/go/interview/1/170050 4490027F1022 $1015 \quad 1 F$

http://www.info.pmda.go.jp/go/interview/2/170050 2149039F1031 $2018 \quad 1 \mathrm{~F}$

http://www.info.pmda.go.jp/go/interview/1/430574 1149019C1149 1 LO8 $1 \mathrm{~F}$

http://www.pmda.go.jp/PmdaSearch/ivakuDetail/ResultDataSetPDF/400256 2149027F1020 109

http://www.info.pmda.go.jp/go/interview/1/170033 3136004C1038 1006 1F

http://www.info.pmda.go.jp/go/interview/1/650168 1149035F1020 $1156 \quad 1 F$

http://www.info.pmda.go.jp/go/interview/1/170033 3160002M2028 1008 1F

http://www.info.pmda.go.jp/go/interview/1/230109 2399009F1149 $1015 \quad 1 \mathrm{~F}$

http://www.info.pmda.go.jp/go/interview/1/270072 2321001F1042 $1002 \quad 1 F$

http://www.info.pmda.go.jp/go/interview/1/650168 2129003M1021 1 14Y 1F http://www.info.pmda.go.jp/go/interview/1/230109 4490026F2040 1035 1F. http://www.info.pmda.go.jp/go/interview/1/400093 2399010B1034 $10201 \mathrm{~F}$ http://www.info.pmda.go.jp/go/interview/1/300119 2149019F1085 1041 1F http://www.info.pmda.go.jp/go/interview/1/400315 2190021B1095 1100 1F http://www.info.pmda.go.jp/go/interview/1/450045 2171017F1028 1009 1F http://www.info.pmda.go.jp/go/interview/1/630004 2171014M1104 $1001 \quad 1 F$ http://www.info.pmda.go.jp/go/interview/3/800126 2149022F1028 $31 \mathrm{~F}$

http://www.pmda.go.jp/PmdaSearch/iyakuDetail/ResultDataSetPDF/3800772325005F1031 106 http://www.info.pmda.go.jp/go/interview/1/530471 1179044F4028 $118 \mathrm{~F} 1 \mathrm{~F}$ http://www.info.pmda.go.jp/go/interview/1/230124 4490025F3026 1006 1F http://www.info.pmda.go.jp/go/interview/1/450045 6250021M1027 $1026 \quad 1 \mathrm{~F}$ http://www.info.pmda.go.jp/go/interview/2/340278 1179041F1025 1018 1F 
Pergolide mesylate

Perindopril erbumine

Pilsicainide hydrochloride hydrate

Pioglitazone hydrochloride

Pranlukast hydrate

Pravastatin sodium

Procaterol hydrochloride hydrate

Propiverine hydrochloride

Quetiapine fumarate

Ranitidine hydrochloride

Rebamipide

Risedronate sodium hydrate

Risperidone

Sarpogrelate hydrochloride

Simvastatin

Sultamicillin tosilate hydrate

Tacrolimus hydrate

Taltirelin hydrate

Tamoxifen citrate

Tegafur

Temocapril hydrochloride

Teprenone

Terbinafine hydrochloride

Ticlopidine hydrochloride

Tocopherol nicotinate

Tulobuterol hydrochloride

Ursodeoxycholic acid

Valacyclovir hydrochloride http://www.info.pmda.go.jp/go/interview/2/230124 1169008F1026 2004 1F

http://www.info.pmda.go.jp/go/interview/3/230124 2144012F1028 $3001 \quad 1 F$

http://www.info.pmda.go.jp/go/interview/3/430574 2129008M1024 3 S10 $1 F$ http://www.info.pmda.go.jp/go/interview/1/4002563969007F3027 1007 1F

http://www.info.pmda.go.jp/go/interview/1/180188 4490017M1036 1009 1F

http://www.info.pmda.go.jp/go/interview/2/430574 2189010C1032 2 M10 $1 F$

http://www.info.pmda.go.jp/go/interview/1/180078 2259004F2168 1005 1F

http://www.info.pmda.go.jp/go/interview/1/400107 2590007C1025 1 04L 1F

http://www.info.pmda.go.jp/go/interview/2/800126 1179042C1023 $2 \quad 1 F$

http://www.info.pmda.go.jp/go/interview/1/340278 2325002F1194 1009 1F

http://www.info.pmda.go.jp/go/interview/1/180078 2329021D1020 $1013 \quad 1 \mathrm{~F}$

http://www.info.pmda.go.jp/go/interview/2/111890 3999019F1026 $2016 \quad 1 F$

http://www.info.pmda.go.jp/go/interview/1/800155 1179038F5029 1005 1F

http://www.info.pmda.go.jp/go/interview/3/400315 3399006C1020 3 15A $1 \mathrm{~F}$

http://www.info.pmda.go.jp/go/interview/2/170050 2189011F1025 $2022 \quad 1 F$

http://www.info.pmda.go.jp/go/interview/2/671450 6131008F1030 2 1F

http://www.info.pmda.go.jp/go/interview/3/800126 3999014D1022 $3 \quad$ 1F

http://www.info.pmda.go.jp/go/interview/1/400315 1190014F2021 $1050 \quad 1 \mathrm{~F}$

http://www.info.pmda.go.jp/go/interview/1/670227 4291003F1163 $1012 \quad 1 \mathrm{~F}$

http://www.info.pmda.go.jp/go/interview/1/400107 4229101F1026 $110 \mathrm{E} \quad 1 \mathrm{~F}$

http://www.info.pmda.go.jp/go/interview/3/430574 2144009F1026 $3 \quad$ C10 $1 F$

http://www.info.pmda.go.jp/go/interview/1/170033 2329012C1026 1009 1F

http://www.info.pmda.go.jp/go/interview/1/730012 6290005F1032 1006 1F

http://www.info.pmda.go.jp/go/interview/4/780069 3399001C1027 4011 1F

http://www.info.pmda.go.jp/go/interview/1/170033 2190006C1037 $1008 \quad 1 \mathrm{~F}$

http://www.info.pmda.go.jp/go/interview/3/100159 2259002F1065 $3004 \quad 1 F$

http://www.info.pmda.go.jp/go/interview/2/400315 2362001F1088 2 11A $1 \mathrm{~F}$

http://www.info.pmda.go.jp/go/interview/1/340278 6250019F1020 $1013 \quad 1 F$ 
Valproate sodium

Valsartan

Voglibose

Zolpidem tartrate

otp.//Www.info.pma.go.jp/go/interview/3/800126 $1129009 F 1025$ 3 $1 F$ (http://www.accessdata.fda.gov/scripts/cder/drugsatfda/index.cfm).

http://www.info.pmda.go.jp/go/interview/1/230124 1139004F1096 1005 1F

http://www.info.pmda.go.jp/go/interview/1/300242 2149041F5026 5 DIO 1F

http://www.info.pmda.go.jp/go/interview/1/400256 3969004F3023 1006 1F

http://www.info.pmda.go.jp/go/interview/3/800126 1129009F1025 3 1F from the FDA website 
Supplement Table 3. Model equation for NC calculation by using the Henderson-Hasselbalch equation.

\section{Compound type}

Mono acid

Mono base

Di acid

Di base

Mono acid/mono base

Tri acid

Tri base

Di acid/mono base

Mono acid/di base

\section{Model equation}

$\mathrm{NC}=\frac{(-1) \times 10^{-p K_{a}}}{10^{-p K_{a}+10^{-p H}}}$

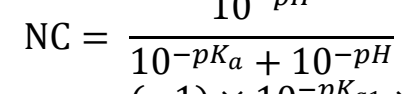

$\mathrm{NC}=\frac{(-1) \times 10^{-p K_{a 1}} \times 10^{-p H}+(-2) \times 10^{-p K_{a 1}} \times 10^{-p K_{a 2}}}{\left[10^{-p H}\right]^{2}+10^{-p K_{a 1}} \times 10^{-p H}+10^{-p K_{a 1}} \times 10^{-p K_{a 2}}}$

$\mathrm{NC}=\frac{2 \times\left[10^{-p H}\right]^{2}+10^{-p K_{a 1}} \times 10^{-p H}}{\left[10^{-p H}\right]^{2}+10^{-p K_{a 1}} \times 10^{-p H}+10^{-p K_{a 1}} \times 10^{-p K_{a 2}}}$

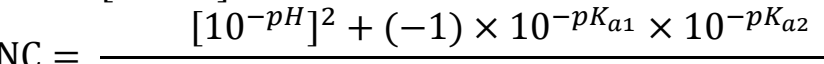

$\mathrm{NC}=\frac{\left[10^{-p H}\right]^{2}+10^{-p K_{a 1}} \times 10^{-p H}+10^{-p K_{a 1}} \times 10^{-p K_{a 2}}}{\left[10^{-1}\right.}$

NC

$=\frac{(-1) \times 10^{-p K_{a 1}} \times\left[10^{-p H}\right]^{2}+(-2) \times 10^{-p K_{a 1}} \times 10^{-p K_{a 2}} \times 10^{-p H}+(-3) \times 10^{-p K_{a 1}} \times 10^{-p K_{a 2}} \times 10^{-p K_{a 3}}}{\left[10^{-p H}\right]^{3}+10^{-p K_{a}} \times\left[10^{-p H}\right)^{2}+10^{-p K_{a 1}} \times 10^{-p K_{a 2}} \times 10^{-p H}+10^{-p a_{a}} \times 10^{-p K_{a 2}} \times 10^{-p K_{a 3}}}$ $\left[10^{-p H}\right]^{3}+10^{-p K_{a 1}} \times\left[10^{-p H}\right]^{2}+10^{-p K_{a 1}} \times 10^{-p K_{a 2}} \times 10^{-p H}+10^{-p K_{a 1}} \times 10^{-p K_{a 2}} \times 10^{-p K_{a 3}}$ $\mathrm{NC}=\frac{3 \times\left[10^{-p H}\right]^{3}+2 \times 10^{-p K_{a 1}} \times\left[10^{-p H}\right]^{2}+10^{-p K_{a 1}} \times 10^{-p K_{a 2}} \times 10^{-p H}}{\left[10^{-p H}\right]^{3}+10^{-p K_{a 1}} \times\left[10^{-p H}\right]^{2}+10^{-p K_{a 1}} \times 10^{-p K_{a 2}} \times 10^{-p H}+10^{-p K_{a 1}} \times 10^{-p K_{a 2}} \times 10^{-p K_{a 3}}}$

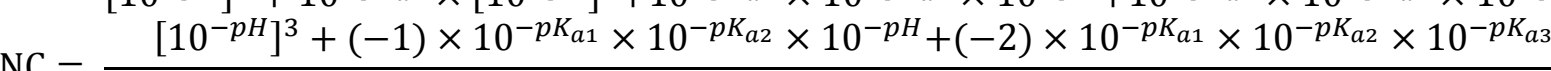
$\mathrm{NC}=\frac{\left.10^{-p H}\right]^{3}+10^{-p K_{a 1}} \times\left[10^{-p H}\right]^{2}+10^{-p K_{a 1}} \times 10^{-p K_{a 2}} \times 10^{-p H}+10^{-p K_{a 1}} \times 10^{-p K_{a 2}} \times 10^{-p K_{a 3}}}{\left[10^{-p H}\right.}$ $\mathrm{NC}=\frac{2 \times\left[10^{-p H}\right]^{3}+10^{-p K_{a 1}} \times\left[10^{-p H}\right]^{2}+(-1) \times 10^{-p K_{a 1}} \times 10^{-p K_{a 2}} \times 10^{-p K_{a 3}}}{\left[10^{-p H}\right]^{3}+10^{-p K_{a 1}} \times\left[10^{-p H}\right]^{2}+10^{-p K_{a 1}} \times 10^{-p K_{a 2}} \times 10^{-p H}+10^{-p K_{a 1}} \times 10^{-p K_{a 2}} \times 10^{-p K_{a 3}}}$ 
Supplement Table 4 Solubility Definitions

\begin{tabular}{llll}
\hline $\begin{array}{l}\text { Solubility } \\
\text { definition }\end{array}$ & $\begin{array}{l}\text { Parts of solvent required for } \mathbf{1} \\
\text { part of solute }\end{array}$ & $\begin{array}{l}\text { Solubility range } \\
(\mathbf{m g} / \mathbf{m L})\end{array}$ & $\begin{array}{l}\text { Solubility assigned } \\
(\mathbf{m g} / \mathbf{m L})\end{array}$ \\
\hline $\begin{array}{l}\text { very soluble } \\
\text { freely soluble }\end{array}$ & $<1$ & $\geq 1000$ & 1000 \\
soluble & $1-10$ & $100-1000$ & 100 \\
sparingly soluble & $30-100$ & $33-100$ & 33 \\
slightly soluble & $100-1000$ & $10-33$ & 10 \\
$\begin{array}{l}\text { very slightly } \\
\text { soluble }\end{array}$ & $1000-10000$ & $1-10$ & 1 \\
$\begin{array}{l}\text { practically } \\
\text { insoluble }\end{array}$ & $\geq 10000$ & $0.1-1$ & 0.1 \\
\hline
\end{tabular}

